TITLE:

\title{
An exact algorithm for the unrestricted block relocation problem
}

\section{$\operatorname{AUTHOR}(\mathrm{S})$ :}

Tanaka, Shunji; Mizuno, Fumitaka

\section{CITATION:}

Tanaka, Shunji ...[et al]. An exact algorithm for the unrestricted block relocation problem. Computers \& Operations Research 2018, 95: 12-31

\section{ISSUE DATE:}

2018-07

URL:

http://hdl.handle.net/2433/236096

\section{RIGHT:}

(c) $<2018>$. This manuscript version is made available under the CC-BY-NC-ND 4.0 license

http://creativecommons.org/licenses/by-nc-nd/4.0/:; The full-text file will be made open to the public on 01 July 2021 in accordance with publisher's 'Terms and Conditions for Self-Archiving'.; This is not the published version. Please cite only the published version.; この論文は出版社版でありません。引用の際には出版社版をご確認ご利用ください。 


\title{
An exact algorithm for the unrestricted block relocation problem*
}

\author{
Shunji Tanaka ${ }^{\mathrm{a}, *}$, Fumitaka Mizuno ${ }^{\mathrm{b}}$ \\ ${ }^{a}$ Institute for Liberal Arts and Sciences, Kyoto University \\ Kyotodaigaku-Katsura, Nishikyo-ku, Kyoto 615-8510, Japan \\ ${ }^{b}$ Department of Electrical Engineering, Kyoto University \\ Kyotodaigaku-Katsura, Nishikyo-ku, Kyoto 615-8510, Japan
}

\begin{abstract}
The purpose of this study is to propose an exact algorithm for the unrestricted block relocation problem with distinct priorities. In this problem, a storage area is considered where blocks of the same size are stacked vertically in tiers. Because we can access only topmost blocks, relocations of blocks are required when other blocks are retrieved. The objective is to minimize the total number of such relocations necessary for retrieving all the blocks one by one according to a specified order. In the restricted version of this problem, only the topmost block above the target block is relocatable. On the other hand, no such restriction is imposed on the unrestricted problem, which is considered in this study. We also assume that each block is assigned a distinct retrieval priority and the retrieval order of blocks is unique. To improve the efficiency of a branch-and-bound algorithm for this problem, we propose several dominance properties to eliminate unnecessary nodes in the search tree. Furthermore, we propose a new lower bound of the total number of relocations. The effectiveness of the proposed exact algorithm is verified by numerical experiments for benchmark instances in the literature.
\end{abstract}

Keywords: block relocation problem, container relocation problem, exact algorithm, dominance properties, lower bound

\footnotetext{
*E-mail: tanaka@kuee.kyoto-u.ac.jp

*Preliminary versions of this study were presented at 2015 IEEE International Conference on Automation Science and Engineering (IEEE CASE 2015), August 2015, and 2015 IEEE International Conference on Industrial Engineering and Engineering Management (IEEM 2015), December 2015.
} 


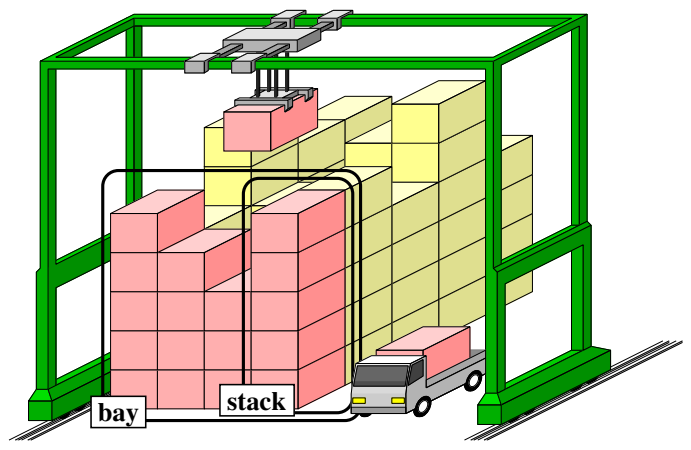

Figure 1: Containers stored in a container yard equipped with a gantry crane

\section{Introduction}

\subsection{Background}

This paper addresses a retrieval problem of stacked items that typically arises in container terminals. Containers that have arrived at a container terminal via sea or land transport are stored temporarily in yards of the container terminal and wait for future transport there. Due to space limitations, containers are stacked vertically as illustrated in Figure 1. Here, each column of containers is referred to as a stack, and a single row of stacks is referred to as a bay. When retrieval of a container is requested, a gantry crane picks it up from a bunch of containers and puts it on a truck for further transport. However, only the topmost containers of the stacks are accessible from the crane, so that containers above the target container should be relocated (reshuffled) to other places beforehand. Such containers are usually relocated to other stacks in the same bay because crane travel across bays is more time-consuming than that within the same bay. To determine destination stacks of the containers, we need to take future retrieval into consideration; otherwise, relocated containers may block the next target container and cause further relocations. Reduction of such unproductive relocations is crucial for improving the throughput of container handling in a sea port. For this reason, the container relocation problem or the block(s) relocation problem has been extensively studied in the literature. For the sake of generality, we refer to a container as a block throughout this paper. Accordingly, our problem is referred to as the block relocation problem, or, simply the BRP.

\subsection{Formal description and classification of the block relocation problem}

The BRP in this study is formally described as follows. Consider a bay composed of $S$ stacks. The $s$-th stack $(1 \leq s \leq S)$ is referred to as stack $s$. In this 


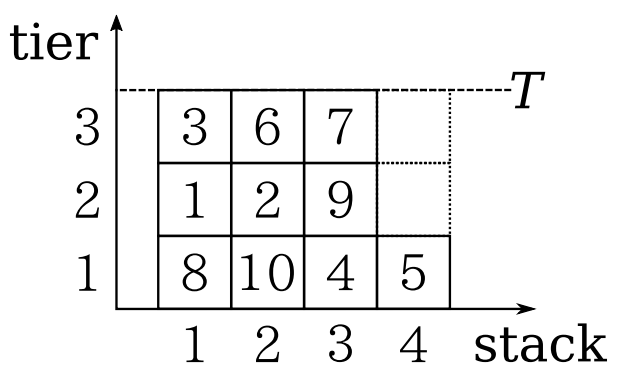

Figure 2: A bay with $S=4, T=3$, and $N=10$. The number in each block is its priority value

bay, $N$ blocks of the same size are stored in tiers. Due to the crane height, the number of blocks in each stack (the stack height) is limited to $T$. Each block is given a unique integer priority value between 1 and $N$, and the block with priority $i$ is referred to as block $i$. An example of a bay with $S=4, T=3$ and $N=10$ is illustrated in Figure 2. All the blocks should be retrieved from the bay one by one in the increasing order of their priorities, which is achieved by the following two types of crane operations:

- Relocation

The topmost block of a stack is moved to the top of another stack whose height does not reach the limit.

- Retrieval

The block to be retrieved next is removed from the bay if it is on the top of a stack.

We need not consider any retrieval explicitly because we can safely assume that blocks are retrieved as soon as they become retrievable. Therefore, a solution is represented by a sequence of relocations. The objective of the BRP is to find a shortest feasible sequence of relocations that retrieves all the blocks from the bay. An example of an optimal solution for the bay configuration in Figure 2 is presented in Figure 3. The total number of relocations is four, and the solution is expressed by the following sequence of relocations: Block 3 is relocated from stack 1 to stack 4, block 7 is relocated from stack 3 to stack 1 , block 6 is relocated from stack 2 to stack 1, and block 9 is relocated from stack 3 to stack 2 .

The BRP can be classified from two aspects. The one is priorities of blocks. In the problem with distinct priorities, all the priority values are different. It follows that the retrieval order is uniquely determined. On the other hand, some blocks 

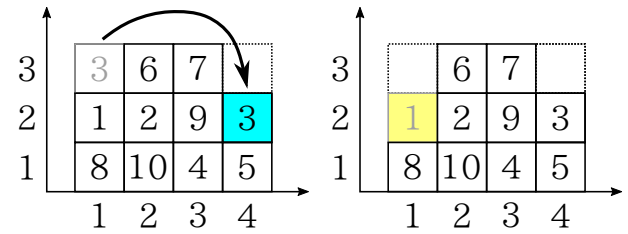

(a) Block 3 is relo-

(b) Block 1 is recated from stack 1 to trieved stack 4
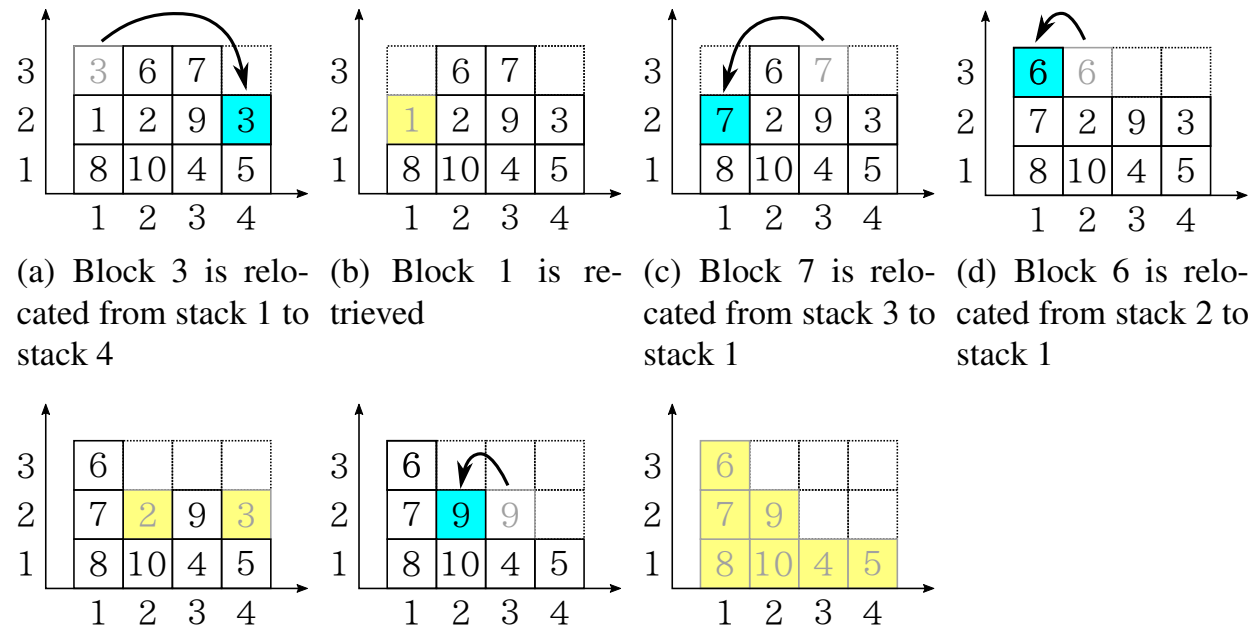

(c) Block 7 is relocated from stack 3 to stack 1

(d) Block 6 is relocated from stack 2 to stack 1

(e) Blocks 2 and 3 (f) Blocks 9 is relo- (g) All the remaining are retrieved cated from stack 3 to blocks are retrieved stack 2

Figure 3: An example of an optimal solution for the bay configuration in Figure 2. The solution is expressed by the following sequence of relocations: (1) block 3 is relocated from stack 1 to stack 4, (2) block 7 is relocated from stack 3 to stack 1, (3) block 6 is relocated from stack 2 to stack 1 , (4) block 9 is relocated from stack 3 to stack 2

are assigned the same priority value in the problem with duplicate priorities, and the retrieval order among them is arbitrary. In other words, the retrieval order is given not among individual blocks but among groups of blocks. The other aspect concerns relocatable blocks. In the unrestricted problem, all topmost blocks are relocatable, while in the restricted problem we can relocate only the topmost block of the stack where the block to be retrieved next is stored. According to this definition, however, it is still unclear which blocks are relocatable in the restricted problem with duplicate priorities, because the block to be retrieved next is not uniquely determined. To avoid this ambiguity, it is assumed that once the block to be retrieved next is determined from those with the highest (smallest) priority, it cannot be changed until it is retrieved.

\subsection{Literature review}

There are rich studies on the BRP and related problems as surveyed by Lehnfeld and Knust [1]. With regard to the complexity of the problem, Blasum et al. [2] proved the NP-completeness of the tram problem that is similar to the BRP with duplicate priorities. This problem aims to retrieve tram cars of different types 
from sidings of a depot in a specified order without any relocations. For the problem of minimizing the total number of relocations, Caserta et al. [3] proved the NP-hardness, following the results by König et al. [4].

Kim and Hong [5] first considered the BRP in the form explained in 1.2. They proposed a depth-first branch-and-bound algorithm and a greedy heuristic for the restricted problem with duplicate priorities. After this research, various heuristic and exact algorithms have been developed. Wan et al. [6] provided a binary ILP formulation and several greedy heuristics for the restricted BRP with distinct priorities. They also considered the dynamic version of the BRP where blocks dynamically arrive at the bay. Caserta et al. [7] proposed a binary matrix representation of bay configurations and constructed a greedy look-ahead heuristic using it. Their heuristic is also referred to as the min-max heuristic. Caserta and Voß [8] and Caserta et al. [9] applied a metaheuristic algorithm called the Corridor Method to the restricted BRP with distinct priorities. Lee and Lee [10] proposed a three-phase heuristic to minimize crane operation time for the restricted BRP with distinct priorities where multiple bays were considered. Ünlüyurt and Aydın [11] proposed a depth-first branch-and-bound algorithm and greedy heuristics for the restricted BRP with distinct priorities. As [10], they considered minimization of crane operation time. Caserta et al. [3] derived binary ILP formulations of the restricted and unrestricted BRPs with distinct priorities, respectively. Forster and Bortfeldt [12] constructed a metaheuristic based on a tree search for the unrestricted BRP with duplicate priorities. Zhu et al. [13] proposed a depth-first iterative deepening $A^{*}$ (IDA*) algorithm as well as several greedy heuristics for the restricted and unrestricted BRPs with distinct priorities. Petering and Hussein [14] proposed an MILP formulation for the unrestricted BRP with distinct priorities. They also proposed the LA-N heuristic by extending the look-ahead heuristic in [7]. Jovanovic and Voß [15] proposed a chain heuristic for the restricted BRP with distinct priorities, which improves the look-ahead heuristic by introducing a heuristic function that takes into account future relocations. Zehendner and Feillet [16] proposed a branch-and-price algorithm for the restricted BRP with distinct priorities. Expósito-Izquierdo et al. [17] applied a best-first A* algorithm for the restricted and unrestricted BRPs with distinct priorities, and proposed a domain-specific knowledge based heuristic for the unrestricted BRP with distinct priorities. The same authors [18] later improved their exact algorithm for the restricted BRP with distinct priorities and corrected a mistake in the formulation of the restricted BRP proposed in [3]. Jin et al. [20] proposed a look-ahead heuristic that employs a tree search for the unrestricted problem with distinct priorities. Zehendner et al. [21] also corrected the mistake in the formulation [3] and 
improved it for the restricted BRP with distinct priorities. Lin et al. [22] proposed a heuristic for the restricted BRP with duplicate priorities. In this problem, they considered multiple bays and assumed a crane with multiple spreaders capable of moving more than one container simultaneously. Eskandari and Azari [19] corrected the formulation in [3] and proposed some cuts to improve it. Tanaka and Takii [23] improved the lower bound in [13] and proposed a depth-first branchand-bound algorithm for the restricted BRP with distinct and duplicate priorities. $\mathrm{Ku}$ and Arthanari [24] proposed another depth-first branch-and-bound algorithm for the restricted BRP with distinct priorities. Recently, Zhang et al [25] considered the unrestricted BRP with distinct priorities that allows batch moves to relocate/retrieve several blocks at a time, and proposed a metaheuristic based on a tree search. After the first submission of this paper, some related papers were published. Tricoire et al. [26] proposed efficient greedy and metaheuristic algorithms for the unrestricted BRP with distinct priorities. They also proposed a new lower bound as a generalization of the lower bound by Forster and Bortfeldt [12], and applied it in their branch-and-bound algorithm. In spite of the fact the new lower bound is always tighter than the lower bound in [12], the average computation time of the branch-and-bound algorithm with the former was almost always longer than that with the latter. de Melo da Silva et al. [27] considered a special class of the BRP with duplicate priorities with two priority values, that is, blocks are composed of only two groups. They also considered a lexicographic bi-objective problem whose secondary objective is the expected number of relocations of the forthcoming retrieval. Galle et al. [28] proposed another ILP formulation of the restricted BRP with distinct priorities based on binary encoding of bay configurations [7]. Their formulation currently yields the best IP approach for this class of problem.

Relocation of items is studied not only as the BRP but also in various forms. In the dynamic or online BRP [29, 30] incoming blocks as well as outgoing blocks are considered as in [6]. A stochastic version of the BRP is also studied in [31]. The ship stowage problem [32-37] also considers incoming and outgoing containers, but assumes that they are transported from ports to ports by a container vessel. When a container is unloaded from the vessel at its destination port, those placed above are unloaded together and then loaded again with containers transported from there. Slabs in place of containers are considered in the slab stack shuffling problem [38-44]. In addition to this difference in blocks, relocations are treated in different ways: Relocated slabs are moved back to the original stack in the same order immediately after the target slab is retrieved, or slabs are relocated at most once (a sufficient number of empty stacks are assumed). The pre-marshalling or 
Table 1: Exact approaches for the BRP in the literature

\begin{tabular}{|c|c|c|c|c|c|}
\hline \multirow{2}{*}{ Reference } & \multirow{2}{*}{ Method } & \multicolumn{2}{|c|}{ Restricted } & \multicolumn{2}{|c|}{ Unrestricted } \\
\hline & & Distinct & Duplicate & Distinct & Duplicate \\
\hline Kim and Hong [5] & $\mathrm{b} \& \mathrm{~b}$ & $\bar{\checkmark}$ & $\bar{\checkmark}$ & & \\
\hline Wan [6] & ILP & $\checkmark$ & & & \\
\hline Ünlüyurt and Aydın [11] & $\mathrm{b} \& \mathrm{~b}$ & $\checkmark$ & & & \\
\hline Caserta et al. [3] & ILP & $\checkmark$ & & $\checkmark$ & \\
\hline Zhu et al. [13] & IDA $^{*}$ & $\checkmark$ & & $\checkmark$ & \\
\hline Petering and Hussein [14] & MILP & & & $\checkmark$ & \\
\hline Zehendner and Feillet [16] & $\mathrm{b} \& \mathrm{p}$ & $\checkmark$ & & & \\
\hline Expósito-Izquierdo et al. [17] & $\mathrm{A}^{*}, \mathrm{ILP}$ & $\checkmark$ & & $\checkmark$ & \\
\hline Expósito-Izquierdo et al. [18] & $\mathrm{b} \& \mathrm{~b}$ & $\checkmark$ & & & \\
\hline Zehendner et al. [21] & ILP & $\checkmark$ & & & \\
\hline Eskandari and Azari [19] & ILP & $\checkmark$ & & & \\
\hline Tanaka and Takii [23] & $\mathrm{b} \& \mathrm{~b}$ & $\checkmark$ & $\checkmark$ & & \\
\hline $\mathrm{Ku}$ and Arthanari [24] & $\mathrm{b} \& \mathrm{~b}$ & $\checkmark$ & & & \\
\hline Tricoire et al. [26] & $\mathrm{b} \& \mathrm{~b}$ & & & $\checkmark$ & \\
\hline de Melo da Silva et al. [27] & $\mathrm{b} \& \mathrm{~b}$ & & $\checkmark^{\dagger}$ & & \\
\hline Galle et al. [28] & ILP & $\checkmark$ & & & \\
\hline This study & $\mathrm{b} \& \mathrm{~b}$ & $\checkmark$ & & $\checkmark$ & \\
\hline
\end{tabular}

re-marshalling problem [45-59] does not consider retrieval operations. It aims to rearrange blocks into a desirable configuration only by relocations. Here, desirable means that no relocations are necessary for retrieving blocks. A similar problem is studied under the name of Blocks World [60, 61].

\subsection{Purpose of this study}

Among the existing studies for the BRP, exact approaches are summarized in Table 1. For the unrestricted BRP with distinct priorities, the ILP and MILP approaches in $[3,14]$ are not competitive with dedicated exact algorithms [13, 17]. In [14], instances with up to only 9 blocks were solved to optimality, while instances with more than 30 blocks were considered in [13, 17]. For the restricted BRP, the branch-and-bound algorithm in [23] seems to be the fastest so far.

The primary purpose of this study is to construct an efficient exact algorithm for the unrestricted BRP with distinct priorities. We also improve our previous exact algorithm [23] for the restricted BRP with distinct priorities. The frame- 
work of the algorithm is the same as those in [13, 23]. Although Zhu et al. [13] called their algorithm an (iterative deepening) $\mathrm{A}^{*}$ algorithm, we prefer a branchand-bound algorithm for describing ours because it traverses the search tree not in best-first but depth-first order. To reduce the size of the search tree in this branchand-bound algorithm, we first propose several dominance properties. These dominance properties tell us that some partial sequences of relocations never appear in an optimal sequence, or, at least an optimal sequence without them exists. Therefore, they enable us to eliminate unnecessary nodes in the search tree. For the pre-marshalling problem, dominance properties have already been proposed and employed $[55,59]$. However, they are not applicable to the BRP directly because the pre-marshalling problem does not take into account retrieval of blocks. This fact motivated us to develop novel dominance properties specific to the BRP. Next, we propose a new lower bound of the total number of relocations based on the lower bound by Forster and Bortfeldt [12]. It also contributes to improving the efficiency of the branch-and-bound algorithm. The effectiveness of the proposed algorithm is demonstrated by computational experiments for benchmark instances in the literature.

The remainder of this paper is organized as follows. First, notation and definitions used throughout this paper are provided in Section 2. Next, an outline of the exact algorithm is presented in Section 3. Then, the dominance properties are derived in Section 4 to eliminate unnecessary nodes in the search tree of the branch-and-bound algorithm. How to ensure their consistency is discussed in Section 5. The new lower bound is proposed in Section 6. In Section 7, the effectiveness of the proposed algorithm is demonstrated by computational experiments. Finally, the results obtained in this study and future research directions are described in Section 8.

\section{Notation and definitions}

In this section we introduce the notation and definitions used throughout this paper. They are summarized in Table 2.

The position of block $i$ in a configuration $\mathscr{C}$ is denoted by $\left(S_{i}(\mathscr{C}), T_{i}(\mathscr{C})\right)$ when it is placed in the $T_{i}(\mathscr{C})$-th tier of stack $S_{i}(\mathscr{C})$. The total number of blocks in stack $s$ and the total number of blocks in the bay are denoted by $N_{s}(\mathscr{C})$ and $N(\mathscr{C})\left(:=\sum_{j=1}^{S} N_{j}(\mathscr{S})\right)$, respectively. The set of indices of stacks is denoted by $\mathscr{S}(:=\{1,2, \ldots, S\})$. The set of indices of slack stacks whose height is lower than $T$ is denoted by $\mathscr{S}^{\text {slack }}(\mathscr{C})\left(:=\left\{s \in \mathscr{S} \mid N_{s}(\mathscr{C})<T\right\}\right)$. The priority of the block in the $t$-th tier of stack $s$ is denoted by $P_{s t}(\mathscr{C})$. Since it is assumed that 
Table 2: Notation and Definitions

\begin{tabular}{|c|c|}
\hline$\left(S_{i}(\mathscr{C}), T_{i}(\mathscr{C})\right)$ & $\begin{array}{l}\text { The position of block } i \text { in } \mathscr{C} \text {. Block } i \text { is stored in the } T_{i}(\mathscr{C}) \text {-th } \\
\text { tier of stack } S_{i}(\mathscr{C}) \text {. }\end{array}$ \\
\hline$N_{s}(\mathscr{C})$ & The number of blocks in stack $s$ in $\mathscr{C}$. \\
\hline$N(\mathscr{C})$ & $\begin{array}{l}\text { The total number of blocks in the bay in } \mathscr{C} . N(\mathscr{C}):= \\
\sum_{j=1}^{S} N_{j}(\mathscr{C}) \text {. }\end{array}$ \\
\hline $\mathscr{S}$ & The set of indices of stacks. $\mathscr{S}:=\{1,2, \ldots, S\}$ \\
\hline $\mathscr{S}^{\text {slack }}(\mathscr{C})$ & $\begin{array}{l}\text { The set of indices of slack stacks in } \mathscr{C} . \mathscr{S}^{\text {slack }}(\mathscr{C}):=\{s \in \\
\left.\mathscr{S} \mid N_{s}(\mathscr{C})<T\right\} \text {. }\end{array}$ \\
\hline$P_{s t}(\mathscr{C})$ & $\begin{array}{l}\text { The priority of the block in the } t \text {-th tier of stack } s \text { in } \mathscr{C} \text {. } \\
\left(S_{P_{s t}(\mathscr{C})}(\mathscr{C}), T_{P_{s t}(\mathscr{C})}(\mathscr{C})\right)=(s, t) \text {. }\end{array}$ \\
\hline$P_{S}^{\mathrm{top}}(\mathscr{C})$ & $\begin{array}{l}\text { The priority of the block on the top of stack } s \text { in } \mathscr{C} \text {. } \\
P_{S}^{\text {top }}(\mathscr{C}):=P_{S N_{s}(\mathscr{C})}(\mathscr{C}) .\end{array}$ \\
\hline$Q_{s}(\mathscr{C})$ & $\begin{array}{l}\text { The priority of stack } s \text { in } \mathscr{C} \cdot Q_{s}(\mathscr{C}):=\min _{1 \leq t \leq N_{s}(\mathscr{C})} P_{s t}(\mathscr{C}) \text { if } \\
N_{s}(\mathscr{C})>0 \text {. Otherwise, } Q_{s}(\mathscr{C}):=\infty \text {. }\end{array}$ \\
\hline$f^{*}(\mathscr{C})$ & $\begin{array}{l}\text { The minimum number of relocations necessary for retrieving } \\
\text { all blocks from } \mathscr{C} \text {. The optimal value for } \mathscr{C} \text {. }\end{array}$ \\
\hline$(i, s, d)$ & The relocation that relocates block $i$ from stack $s$ to stack $d$. \\
\hline$L_{i s d}$ & $\begin{array}{l}\text { The relocation mapping. The configuration obtained by ap- } \\
\text { plying }(i, s, d) \text { to } \mathscr{C} \text { is given by } L_{i s d}(\mathscr{C}) \text {. }\end{array}$ \\
\hline$R$ & $\begin{array}{l}\text { The retrieval mapping. The configuration obtained by remov- } \\
\text { ing all retrievable blocks from } \mathscr{C} \text { is given by } R(\mathscr{C}) \text {. }\end{array}$ \\
\hline target block & $\begin{array}{l}\text { The block to be retrieved next. The block with the highest } \\
\text { (smallest) priority in the bay. }\end{array}$ \\
\hline blocking block & $\begin{array}{l}\text { A block under which a block with a higher (smaller) } \\
\text { priority is placed. Block } i \text { is a blocking block if } i> \\
\min _{1 \leq t<T_{i}(\mathscr{C})} P_{S_{i}(\mathscr{C}) t}(\mathscr{C}) .\end{array}$ \\
\hline $\mathrm{BB}$ relocation & A relocation $(i, s, d)$ such that $i>Q_{s}(\mathscr{C}) \wedge i>Q_{d}(\mathscr{C})$. \\
\hline $\mathrm{BG}$ relocation & A relocation $(i, s, d)$ such that $i>Q_{s}(\mathscr{C}) \wedge i<Q_{d}(\mathscr{C})$. \\
\hline GB relocation & A relocation $(i, s, d)$ such that $i<Q_{s}(\mathscr{C}) \wedge i>Q_{d}(\mathscr{C})$. \\
\hline GG relocation & A relocation $(i, s, d)$ such that $i<Q_{s}(\mathscr{C}) \wedge i<Q_{d}(\mathscr{C})$. \\
\hline$i \in \mathscr{C}$ & Block $i$ is present in $\mathscr{C}$ \\
\hline $\mathscr{C}_{\mathrm{a}} \leq \mathscr{C}_{\mathrm{b}}$ & $\mathscr{C}_{\mathrm{a}}$ dominates $\mathscr{C}_{\mathrm{b}}$. \\
\hline
\end{tabular}


block $i$ has priority $i$, we have $\left(S_{P_{s t}(\mathscr{C})}(\mathscr{C}), T_{P_{s t}(\mathscr{C})}(\mathscr{C})\right)=(s, t)$. The priority of the topmost block of stack $s$ is denoted by $P_{S}^{\text {top }}(\mathscr{C})\left(:=P_{s N_{s}(\mathscr{C})}(\mathscr{C})\right)$. The highest (smallest) priority in stack $s$ is referred to as the priority of stack $k$ and denoted by $Q_{s}(\mathscr{C})\left(:=\min _{1 \leq t \leq N_{s}(\mathscr{C})} P_{s t}(\mathscr{C})\right)$. If stack $s$ is empty, $Q_{s}(\mathscr{C})$ is defined by $Q_{s}(\mathscr{C}):=\infty$. The minimum number of relocations necessary for retrieving all blocks from $\mathscr{C}$ is denoted by $f^{*}(\mathscr{C})$. As long as the configuration $\mathscr{C}$ currently considered is obvious, $(\mathscr{C})$ is omitted in the above notations: $S_{i}$ is used in place of $S_{i}(\mathscr{C}), N_{s}$ in place of $N_{s}(\mathscr{C})$, and so on.

An operation of relocating block $i$ from stack $s$ to stack $d(\neq s)$ is denoted by a triplet $(i, s, d)$. It is also denoted by a mapping $L_{i s d}$ defined over a set of configurations: The new configuration obtained by applying relocation $(i, s, d)$ to $\mathscr{C}$ is given by $L_{i s d}(\mathscr{C})$. The new configuration obtained by removing all retrievable blocks one by one from $\mathscr{C}$ is denoted using a mapping $R$ as $R(\mathscr{C})$. Configuration $\mathscr{C}$ is said to be minimal when no blocks can be retrieved from $\mathscr{C}$, that is, $\mathscr{C}=R(\mathscr{C})$.

The block with the highest (smallest) priority in the bay is referred to as the target block. The target block is the block to be retrieved next. Block $i$ is said to be a blocking block if $i>\min _{1 \leq t<T_{i}} P_{S_{i} t}$, that is, a block with a higher (smaller) priority is placed under it. According to [12], a relocation is classified into $\mathrm{BB}, \mathrm{BG}$, $\mathrm{GB}$, and GG relocations. The first $\mathrm{B}$ or $\mathrm{G}$ means whether the relocated block is a blocking block (B) or not $(\mathrm{G})$, and the second $\mathrm{B}$ or $\mathrm{G}$ means whether it becomes a blocking block after relocation (B) or not $(\mathrm{G})$.

If block $i$ is present in $\mathscr{C}$, we write $i \in \mathscr{C}$. Relocation $(i, s, d)$ is feasible for a minimal $\mathscr{C}$ if $i \in \mathscr{C}, S_{i}(\mathscr{C})=s, T_{i}(\mathscr{C})=N_{s}(\mathscr{C})$, and $N_{d}(\mathscr{C})<T$ are all satisfied. Relocation $(i, s, d)$ is admissible for a minimal $\mathscr{C}$ if either it is feasible for $\mathscr{C}$ or $i \notin \mathscr{C}$ holds. For ease of notation, $L_{i s d}(\mathscr{C})$ is defined by $L_{i s d}(\mathscr{C}):=\mathscr{C}$ for $i \notin \mathscr{C}$. Relocation $(i, s, d)$ is infeasible for a minimal $\mathscr{C}$ when it is not admissible for $\mathscr{C}$.

A (partial) solution of the BRP is given by a sequence of relocations $\left(i_{1}, s_{1}, d_{1}\right)$, $\left(i_{2}, s_{2}, d_{2}\right), \ldots,\left(i_{n}, s_{n}, d_{n}\right)$. We say that this sequence is feasible for a minimal $\mathscr{C}$ if, for any $k=1, \ldots, n$, relocation $\left(i_{k}, s_{k}, d_{k}\right)$ is feasible for configuration $\mathscr{C}_{k-1}$ where $\mathscr{C}_{k}:=R \circ L_{i_{k} s_{k}} d_{k}\left(\mathscr{C}_{k-1}\right)$ and $\mathscr{C}_{0}:=\mathscr{C}$. Similarly, it is admissible if, for any $k=1$, $\ldots, n$, relocation $\left(i_{k}, s_{k}, d_{k}\right)$ is admissible for configuration $\mathscr{C}_{k-1}$. In addition, it is infeasible if it is not admissible.

A configuration $\mathscr{C}_{\mathrm{a}}$ dominates a configuration $\mathscr{C}_{\mathrm{b}}$ or $\mathscr{C}_{\mathrm{a}} \leq \mathscr{C}_{\mathrm{b}}$, if both the following conditions are satisfied:

(i) $i \in \mathscr{C}_{\mathrm{b}}$ and $S_{i}\left(\mathscr{C}_{\mathrm{b}}\right)=S_{i}\left(\mathscr{C}_{\mathrm{a}}\right)$ hold for any $i \in \mathscr{C}_{\mathrm{a}}$,

(ii) $T_{i}\left(\mathscr{C}_{\mathrm{b}}\right)<T_{j}\left(\mathscr{C}_{\mathrm{b}}\right)$ holds for any $i, j \in \mathscr{C}_{\mathrm{a}}$ satisfying $S_{i}\left(\mathscr{C}_{\mathrm{a}}\right)=S_{j}\left(\mathscr{C}_{\mathrm{a}}\right)$ and 


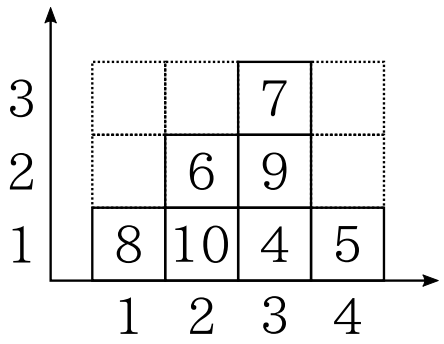

Figure 4: A configuration dominating the configuration in Figure 2. Blocks 1-3 are missing, but the other blocks are placed as in Figure 2

$$
T_{i}\left(\mathscr{C}_{\mathrm{a}}\right)<T_{j}\left(\mathscr{C}_{\mathrm{a}}\right) .
$$

This definition implies that if $\mathscr{C}_{\mathrm{a}} \leq \mathscr{C}_{\mathrm{b}}$ holds, $\mathscr{C}_{\mathrm{a}}$ is the same configuration as $\mathscr{C}_{\mathrm{b}}$ except for blocks $i$ with $i \notin \mathscr{C}_{\mathrm{a}}$ and $i \in \mathscr{C}_{\mathrm{b}}$. Figure 4 illustrates an example of a configuration dominating the configuration in Figure 2.

First, we will give the following two lemmas.

Lemma 1. Consider two configurations $\mathscr{C}_{\mathrm{a}}$ and $\mathscr{C}_{\mathrm{b}}$ satisfying $\mathscr{C}_{\mathrm{a}} \leq \mathscr{C}_{\mathrm{b}}$. Then, $R\left(\mathscr{C}_{\mathrm{a}}\right) \leq R\left(\mathscr{C}_{\mathrm{b}}\right)$ holds

Proof. Obvious.

Lemma 2. Consider two minimal configurations $\mathscr{C}_{\mathrm{a}}$ and $\mathscr{C}_{\mathrm{b}}$ satisfying $\mathscr{C}_{\mathrm{a}} \leq \mathscr{C}_{\mathrm{b}}$. If a relocation $(i, s, d)$ is feasible for $\mathscr{C}_{\mathrm{b}}$, it is admissible for $\mathscr{C}_{\mathrm{a}}$ and $L_{i s d}\left(\mathscr{C}_{\mathrm{a}}\right) \leq$ $L_{\text {isd }}\left(\mathscr{C}_{\mathrm{b}}\right)$ holds.

Proof. If $i \in \mathscr{C}_{\mathrm{a}}$, block $i$ should be on the top of stack $s$ in $\mathscr{C}_{\mathrm{a}}$. Otherwise, there exists a block $j$ that satisfies $S_{j}\left(\mathscr{C}_{\mathrm{a}}\right)=s$ and $T_{i}\left(\mathscr{C}_{\mathrm{a}}\right)<T_{j}\left(\mathscr{C}_{\mathrm{a}}\right)$. This block should also be above block $i$ in stack $s$ in $\mathscr{C}_{\mathrm{b}}$ from the definition of $\mathscr{C}_{\mathrm{a}} \leq \mathscr{C}_{\mathrm{b}}$, so that relocation $(i, s, d)$ is infeasible for $\mathscr{C}_{\mathrm{b}}$. However, it contradicts the feasibility of relocation $(i, s, d)$ for $\mathscr{C}_{\mathrm{b}}$. Thus $S_{i}\left(\mathscr{C}_{\mathrm{a}}\right)=s$ and $T_{i}\left(\mathscr{C}_{\mathrm{a}}\right)=N_{s}\left(\mathscr{C}_{\mathrm{a}}\right)$ hold if $i \in \mathscr{C}_{\mathrm{a}}$. Furthermore, $N_{d}\left(\mathscr{C}_{\mathrm{a}}\right) \leq N_{d}\left(\mathscr{C}_{\mathrm{b}}\right)<T$ holds from $\mathscr{C}_{\mathrm{a}} \leq \mathscr{C}_{\mathrm{b}}$ and the feasibility of relocation $(i, s, d)$ for $\mathscr{C}_{\mathrm{b}}$. Therefore, relocation $(i, s, d)$ is feasible for $\mathscr{C}_{\mathrm{a}}$ if $i \in$ $\mathscr{C}_{\mathrm{a}}$, and $L_{i s d}\left(\mathscr{C}_{\mathrm{a}}\right) \leq L_{i s d}\left(\mathscr{C}_{\mathrm{b}}\right)$ holds in this case. If $i \notin \mathscr{C}_{\mathrm{a}}$, relocation $(i, s, d)$ is admissible for $\mathscr{C}_{\mathrm{a}}$ and $L_{i s d}\left(\mathscr{C}_{\mathrm{a}}\right)=\mathscr{C}_{\mathrm{a}}$ from the definition of $L_{i s d}$. Thus, $L_{i s d}\left(\mathscr{C}_{\mathrm{a}}\right) \leq$ $L_{i s d}\left(\mathscr{C}_{\mathrm{b}}\right)$ holds also in this case.

Corollary 1. $f^{*}\left(\mathscr{C}_{\mathrm{a}}\right) \leq f^{*}\left(\mathscr{C}_{\mathrm{b}}\right)$ holds if two minimal configurations $\mathscr{C}_{\mathrm{a}}$ and $\mathscr{C}_{\mathrm{b}}$ satisfy $\mathscr{C}_{\mathrm{a}} \leq \mathscr{C}_{\mathrm{b}}$. 

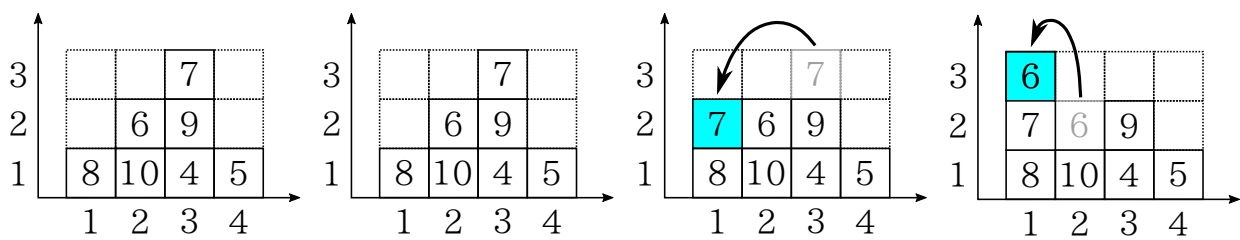

(a) $(3,1,4)$ (no block (b) No block is reis relocated) trieved

(c) $(7,3,1)$

(d) $(6,2,1)$

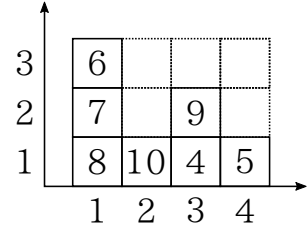

(e) No block is retrieved

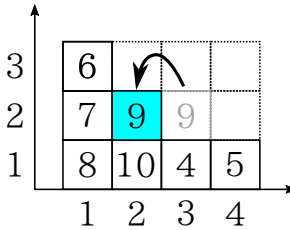

(f) $(9,3,2)$

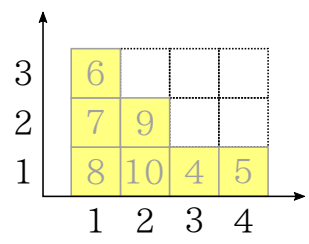

(g) All the remaining blocks are retrieved

Figure 5: Sequence $(3,1,4),(7,3,1),(6,2,1),(9,3,2)$ in Figure 3 applied to the configuration in Figure 4

Proof. From Lemmas 1 and 2, an optimal sequence for $\mathscr{C}_{\mathrm{b}}$ is admissible for $\mathscr{C}_{\mathrm{a}}$.

Figure 5 confirms that the optimal sequence $(3,1,4),(7,3,1),(6,2,1),(9,3,2)$ in Figure 3 for the configuration in Figure $2\left(\mathscr{C}_{\mathrm{b}}\right)$ is admissible for the configuration in Figure $4\left(\mathscr{C}_{\mathrm{a}}\right)$, which dominates $\mathscr{C}_{\mathrm{b}}$. We note that every intermediate configuration in Figure 5 dominates the corresponding configuration in Figure 3 as Lemmas 1 and 2 claim. In this case, a sequence $(7,3,1),(6,2,1),(9,3,2)$ with only three relocations is feasible for $\mathscr{C}_{\mathrm{a}}$ and $f^{*}\left(\mathscr{C}_{\mathrm{a}}\right)<f^{*}\left(\mathscr{C}_{\mathrm{b}}\right)$ holds.

Suppose that a feasible sequence of $n_{\mathrm{b}}$ relocations for a minimal $\mathscr{C}$ yields $\mathscr{C}_{\mathrm{b}}$ and there exists another admissible sequence of $n_{\text {a }}$ relocations for $\mathscr{C}$ that yields $\mathscr{C}_{\mathrm{a}}$. If $n_{\mathrm{a}} \leq n_{\mathrm{b}}$ and $\mathscr{C}_{\mathrm{a}} \leq \mathscr{C}_{\mathrm{b}}, f^{*}(\mathscr{C}) \leq n_{\mathrm{a}}+f^{*}\left(\mathscr{C}_{\mathrm{a}}\right) \leq n_{\mathrm{b}}+f^{*}\left(\mathscr{C}_{\mathrm{b}}\right)$ holds from Corollary 1 . Thus the former sequence is not better than the latter, and we say that the former sequence is dominated by the latter sequence, or, the latter dominates the former. If $n_{\mathrm{a}}<n_{\mathrm{b}}$, the former sequence is never optimal. In this case, we say that the former is strictly dominated by the latter.

\section{Exact algorithm}

In this section we describe an outline of the proposed exact algorithm. 


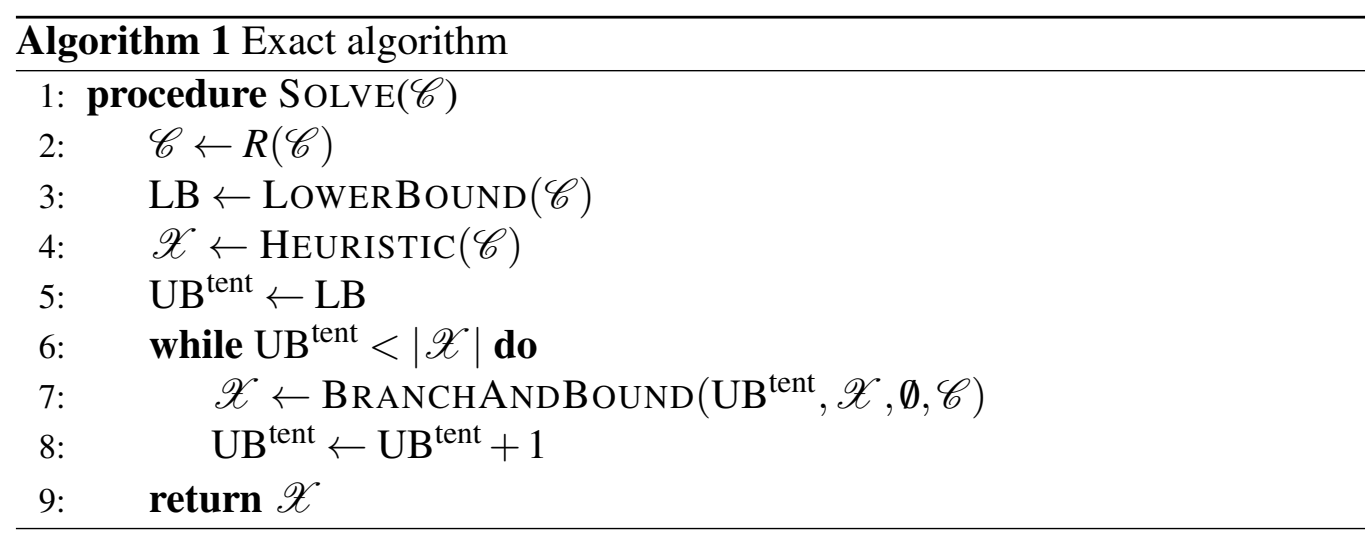

\subsection{Outline of algorithm}

The framework is basically the same as those in [13, 23]. The main loop of the exact algorithm is described as Algorithm 1. It first computes the initial lower bound LB (line 3), and next initializes the current best solution $\mathscr{X}$ by a greedy heuristic explained later (line 4). Then, the tentative upper bound UB ${ }^{\text {tent }}$ is initialized by LB (line 5), and the algorithm searches for a solution whose objective value is equal to $\mathrm{UB}^{\text {tent }}$ by a branch-and-bound algorithm (line 7). If such a solution is found, the algorithm returns $\mathscr{X}$ as an optimal solution and terminates (lines 6 and 9). Otherwise, the optimal value is greater than $\mathrm{UB}^{\text {tent }}$. Hence, UB ${ }^{\text {tent }}$ is increased by one (line 8) and the branch-and-bound algorithm is applied again.

In the branch-and-bound algorithm, an optimal sequence of relocations is searched for from first to last. Thus, a node at depth $k$ in the search tree represents a configuration reached by $k$ relocations from the initial configuration. An example of the search tree is provided in Figure 6. We note that the same configuration as the initial one is obtained by sequence $(3,2,3),(3,3,2)$ : relocating block 3 from stack 2 to stack 3 and then stack 3 to stack 2 . Obviously, such a (partial) sequence is never optimal. In other words, sequence $(3,2,3),(3,3,2)$ is strictly dominated, and we can eliminate the node. We also note that two sequences $(6,1,3),(3,2,1)$ and $(6,1,3),(3,2,3)$ (sequences $(3,2,1)$ and $(3,2,3)$ in practice) yield the same configuration. In this case, each of the two sequences is dominated by the other, and we can eliminate either node, but not both. We will first derive several dominance properties in Section 4 to detect such a dominated sequence and eliminate the corresponding node. Since some of these properties only tell us whether a sequence is dominated or not, we will explain in Section 5 which of the two sequences $(3,2,1)$ and $(3,2,3)$ is adopted as well as how to ensure consistency among the dominance properties. 


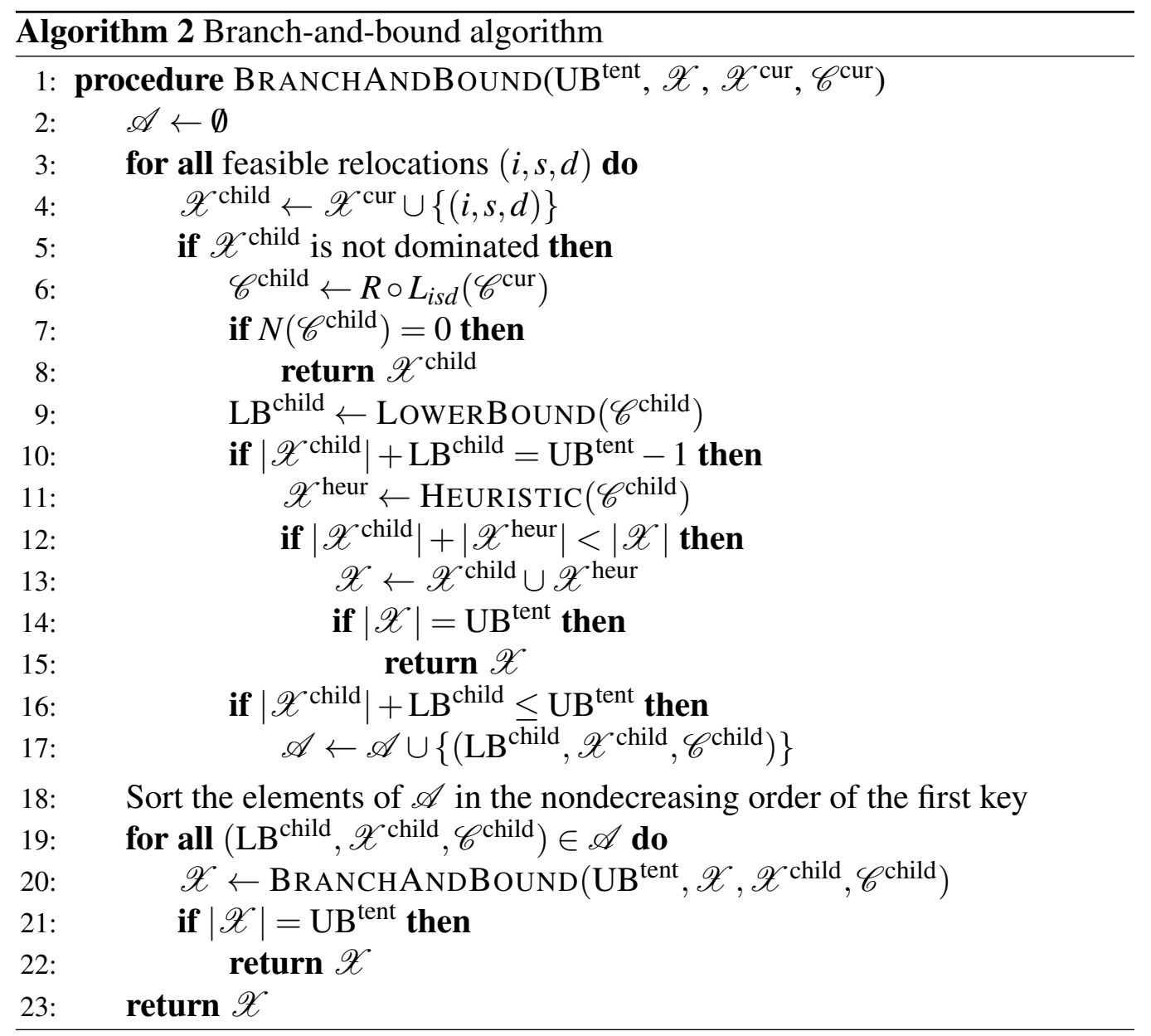




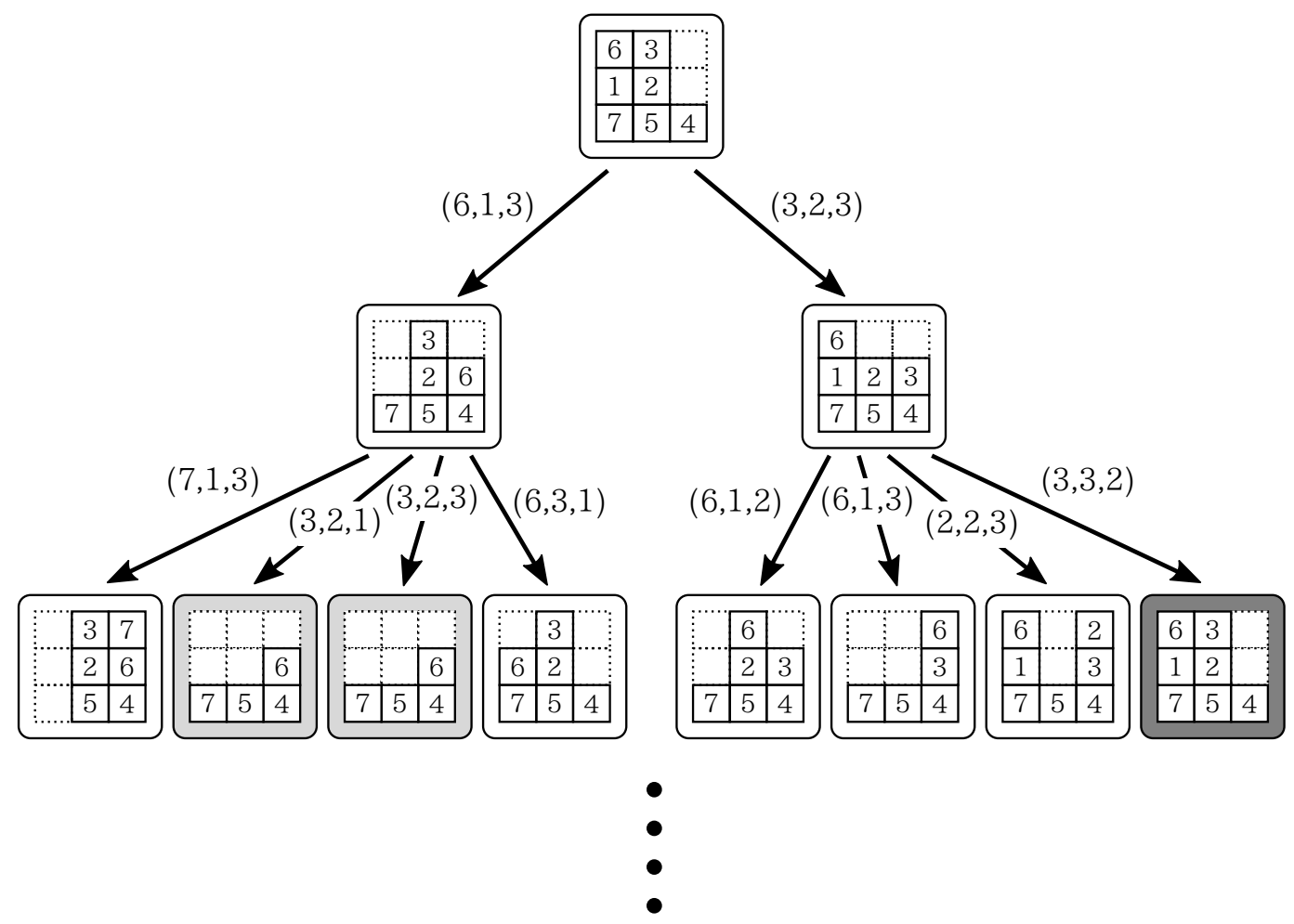

Figure 6: An example of the search tree in the branch-and-bound algorithm

In the branch-and-bound algorithm, Algorithm 2 is called recursively, where $\mathscr{X}$ is the current best sequence of relocations, $\mathscr{X}^{\text {cur }}$ the partial sequence of relocations, and $\mathscr{C}^{\text {cur }}$ the configuration obtained by $\mathscr{X}^{\text {cur }}$. To branch from the current node, all feasible relocations for $\mathscr{C}^{\text {cur }}$ are generated (line 3). Each of them is appended to $\mathscr{X}^{\text {cur }}$ (line 4 ) in order to obtain a sequence $\mathscr{X}^{\text {child }}$ for a child node. Then, whether it is dominated or not is checked (line 5). More specifically, the existence of a dominating sequence for every subsequence of $\mathscr{X}^{\text {child }}$ ending at its last element is checked to see whether $\mathscr{X}^{\text {child }}$ can be eliminated or not. Unless $\mathscr{X}^{\text {child }}$ is dominated, the corresponding configuration $\mathscr{C}^{\text {child }}$ is also generated (line 6). If no block is left in $\mathscr{C}^{\text {child }}$ (line 7), $\mathscr{X}^{\text {child }}$ is a feasible solution, so that the algorithm returns it (line 8). Otherwise, a lower bound is computed (line 9). Only child nodes whose lower bound is less than the current upper bound are added to list $\mathscr{A}$ (lines 16-17). To improve the current best solution, the greedy heuristic in 3.2 is applied to $\mathscr{C}^{\text {child }}$ when $\left|\mathscr{X}^{\text {child }}\right|+\mathrm{LB}^{\text {child }}=\mathrm{UB}^{\text {tent }}-1$ (lines $\left.10-15\right)$. This condition aims to: 
1. filter out unpromising child nodes with a large lower bound,

2. avoid applying the heuristic to the same node more than once.

Suppose that the heuristic is applied to the nodes whose lower bound is small enough to satisfy $\left|\mathscr{X}^{\text {child }}\right|+\mathrm{LB}^{\text {child }} \leq \mathrm{UB}^{\text {tent }}-K$ for some fixed integer $K$. Because the branch-and-bound algorithm is repeatedly employed with $\mathrm{UB}^{\text {tent }}$ increased, the heuristic should have already been applied to the same nodes at a previous iteration if $\left|\mathscr{X}^{\text {child }}\right|+\mathrm{LB}^{\text {child }} \leq\left(\mathrm{UB}^{\text {tent }}-1\right)-K$. It follows that we only need to consider nodes with $\left|\mathscr{X}^{\text {child }}\right|+\mathrm{LB}^{\text {child }}=\mathrm{UB}^{\text {tent }}-K$, and $K$ is chosen as 1 in the algorithm. After child nodes at depth $(k+1)$ are generated, they are traversed one by one (lines 19-22). Thus the depth-first strategy is adopted in this branch-and-bound algorithm. Among the nodes at depth $(k+1)$, the one with the smallest lower bound is traversed first (line 18). When a solution whose objective value is equal to $\mathrm{UB}^{\text {tent }}$ is found, the algorithm immediately terminates (lines 14 and 15, and lines 21 and 22).

\subsection{Greedy heuristic}

The heuristic used for computing upper bounds is a slightly simplified version of PU2 in [13]. This heuristic determines the source and destination stacks of the next relocation as follows:

1. Determine the source stack $s$. Let $s^{\mathrm{T}}:=\arg \min _{j \in \mathscr{S}} Q_{j}$ (the target block is placed in stack $s^{\mathrm{T}}$ ).

(a) If a BG relocation exists for the topmost block $P_{s^{\mathrm{T}}}^{\text {top }}$ of stack $s^{\mathrm{T}}, s:=s^{\mathrm{T}}$.

(b) If no BG relocation exists for block $P_{s^{\mathrm{T}}}^{\text {top }}$, but a GG relocation of another topmost block $P_{s^{\prime}}^{\text {top }}$ enables it, $s:=s^{\prime}$. If there is more than one candidate for stack $s^{\prime}$, the stack with the largest $N_{s^{\prime}}$ is chosen.

(c) Otherwise, $s:=s^{\mathrm{T}}$.

2. Determine the destination stack $d$.

(a) If a BG or GG relocation exists for block $P_{s}^{\text {top }}$, the stack $d$ with the highest (smallest) stack priority $Q_{d}$ is chosen among those satisfying $P_{s}^{\text {top }}<Q_{d}$.

(b) If no BG or GG relocation exists for block $P_{s}^{\text {top }}$, let stacks $d^{\prime}$ and $d^{\prime \prime}$ be the slack stacks with the lowest (largest) and the second lowest (second largest) stack priorities, respectively.

(i) If $N_{d^{\prime}}=T-1$ and stack $d^{\prime \prime}$ does exist, $d:=d^{\prime \prime}$.

(ii) Otherwise, $d:=d^{\prime}$. 


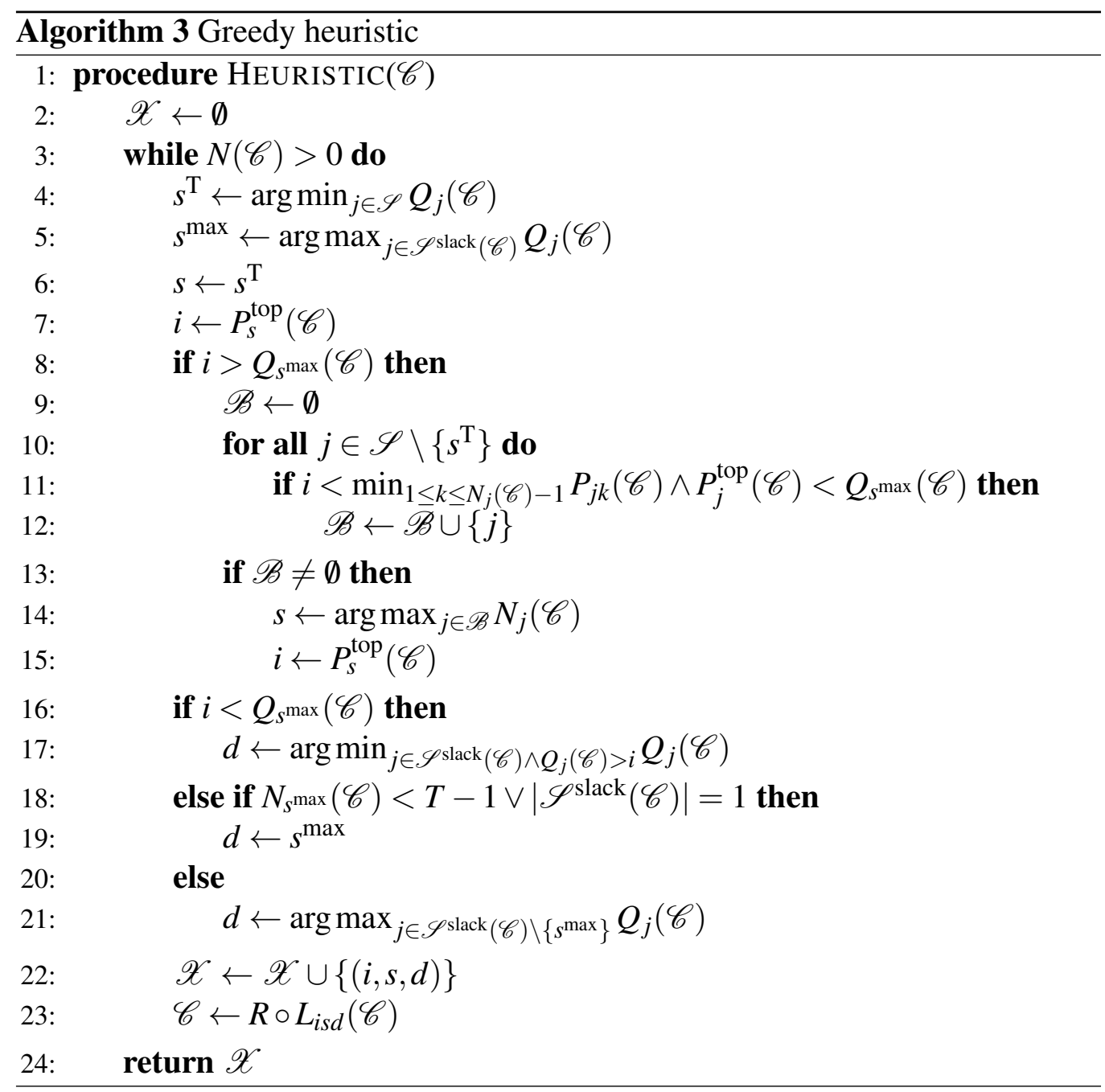


In this procedure, 2(a) chooses the destination stack that minimizes the change of the stack priority. On the other hand, 2(b) chooses the stack that possibly delays future relocations of block $P_{s}^{\text {top }}$. 2(b)(i) tries not to fill stack $d^{\prime}$ in order to accept a $\mathrm{BG}$ or $\mathrm{GG}$ relocation in the future.

Algorithm 3 describes the heuristic in more detail. The source stack and thus the block to be relocated is determined on lines 6-15, and its destination stack is determined on lines 16-21. The algorithm first tries to relocate $P_{s^{\mathrm{T}}}^{\text {top }}$ (lines 6 and 7). If $P_{s^{\mathrm{T}}}^{\mathrm{top}}>Q_{s^{\max }}=\max _{j \in \mathscr{S} \text { slack }} Q_{j}$ holds (line 8), no BG relocation exists for this block. In this case, the algorithm checks whether a GG relocation of the topmost block in a stack $j \neq s^{T}$ enables it (lines 9-12). If such a block is found, it is relocated where ties are broken by the stack height (lines 13-15). Otherwise, block $P_{s}^{\text {top }}$ is relocated. Next, the destination stack of the block (block $i$ ) is determined. If a BG or GG relocation exists for this block (line 16), the stack with the highest (smallest) priority is chosen, as long as the relocation remains $\mathrm{BG}$ or GG, respectively (line 17). If no BG or GG relocation exists, the stack with the lowest (largest) priority is chosen, as long as this relocation does not make the stack full (lines 18-19). If it makes that stack full, the stack with the second lowest (largest) priority is chosen (line 21).

\section{Dominance properties}

As presented in the preceding section, the proposed branch-and-bound algorithm checks dominance properties (line 5 in Algorithm 2) to suppress generation of unnecessary nodes in the search tree. A simple example is sequence $(3,2,3)$, $(3,3,2)$ in Figure 6, which relocates block 3 from stack 2 to stack 3 and immediately relocates it back to stack 2 . Because the same configuration as the original one is generated, we need not consider this sequence in the search tree. In other words, this sequence is dominated. Some dominance properties have already been proposed for the pre-marshalling problem $[55,59]$. However, they are not applicable directly to our problem due to existence of block retrieval. Indeed, the above example is not always true for the BRP: Sequence $(6,1,3),(6,3,1)$ is not dominated in Figure 6 because block 1 is retrieved after relocation $(6,1,3)$. In this section, we propose several dominance properties specific to the unrestricted BRP that take into account retrieval of blocks appropriately. Some of them are also applicable to the restricted BRP, and we employ them to improve our previous algorithm [23].

The dominance properties are classified into three types. Theorems 1-3 concern a pair of transitive relocations. Theorems 1 and 2 assert that a pair of reloca- 
tions from stack A to stack B and from stack B to stack $\mathrm{C}$ can be combined into one relocation from stack A to stack $\mathrm{C}$, whereas Theorem 3 claims that the pair can be replaced by another pair from stack A to stack D and from stack D to stack C. Theorems 4 and 5 are for a pair of independent relocations. If block $\mathrm{X}$ is relocated from stack A to stack B and then block Y is relocated from stack $\mathrm{C}$ to stack $\mathrm{D}$, their order can be interchanged. The last two theorems, Theorems 6 and 7 are on retrieval of a block. If block $\mathrm{X}$ is retrieved after it is relocated from stack $\mathrm{A}$ to stack B, it need not be relocated (Theorem 6), or, it can be relocated to another stack $\mathrm{C}$ (Theorem 7). In the following, a feasible sequence $\left(i_{1}, s_{1}, d_{1}\right),\left(i_{2}, s_{2}, d_{2}\right)$, $\ldots,\left(i_{n}, s_{n}, d_{n}\right)$ for a minimal configuration $\mathscr{C}$ is assumed, and $\mathscr{C}_{k}(k=0, \ldots, n)$ are defined by

$$
\mathscr{C}_{k}:= \begin{cases}R \circ L_{i_{k} s_{k} d_{k}}\left(\mathscr{C}_{k-1}\right), & 1 \leq k \leq n, \\ \mathscr{C}, & k=0 .\end{cases}
$$

Our goal is to provide conditions for the existence of a sequence dominating sequence $\left(i_{1}, s_{1}, d_{1}\right),\left(i_{2}, s_{2}, d_{2}\right), \ldots,\left(i_{n}, s_{n}, d_{n}\right)$.

\subsection{Dominance properties for transitive relocations}

First, three dominance properties on a pair of transitive relocations are provided.

Theorem 1. (Transitive Relocation Rule A) Sequence $\left(i_{2}, s_{2}, d_{2}\right), \ldots,\left(i_{n-1}, s_{n-1}, d_{n-1}\right)$, $\left(i_{1}, s_{1}, d_{n}\right)$ or $\left(i_{2}, s_{2}, d_{2}\right), \ldots,\left(i_{n-1}, s_{n-1}, d_{n-1}\right)$ strictly dominates sequence $\left(i_{1}, s_{1}, d_{1}\right)$, $\left(i_{2}, s_{2}, d_{2}\right), \ldots,\left(i_{n}, s_{n}, d_{n}\right)$ if all the following conditions are satisfied:

(TA1) $s_{n}=d_{1}, i_{n}=i_{1}$,

(TA2) $i_{1} \notin\left\{i_{2}, \ldots, i_{n-1}\right\}$,

(TA3) $s_{1} \notin\left\{s_{2}, d_{2}, \ldots, s_{n-1}, d_{n-1}\right\}$,

(TA4) $N_{s_{1}}\left(\mathscr{C}_{n-1}\right)=N_{s_{1}}(\mathscr{C})-1$.

Proof. First, suppose that $d_{n} \neq s_{1}$. From (TA3) and (TA4), stack $s_{1}$ is not affected by sequence $\left(i_{1}, s_{1}, d_{1}\right), \ldots,\left(i_{n-1}, s_{n-1}, d_{n-1}\right)$ for $\mathscr{C}$ after block $i_{1}$ is relocated to stack $d_{1}$. The configuration of stack $s_{1}$ in $\mathscr{C}_{1}, \ldots, \mathscr{C}_{n-1}$ is the same as that in $\mathscr{C}$ except the absence of block $i_{1}$. It follows that block $i_{1}$ does not interfere any retrieval caused by this sequence for $\mathscr{C}$, even if it is not relocated to stack $d_{1}$. Furthermore, from (TA2), block $i_{1}$ is not relocated by sequence $\left(i_{2}, s_{2}, d_{2}\right)$, 


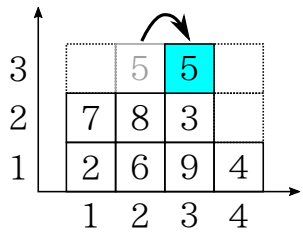

(a) $(5,2,3)$

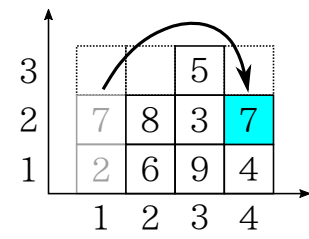

(b) $(7,1,4)$

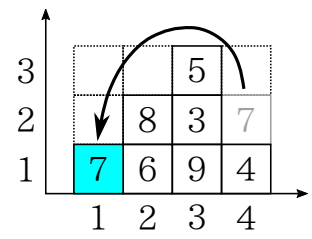

(c) $(7,4,1)$

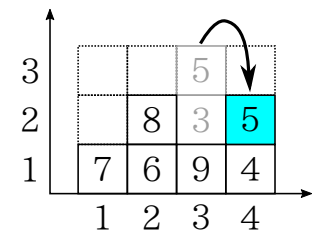

(d) $(5,3,4)$

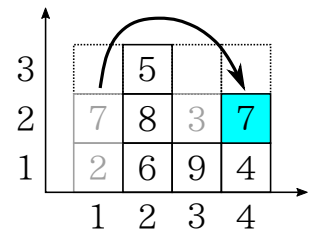

(e) $(7,1,4)$

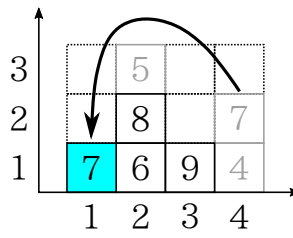

(f) $(7,4,1)$

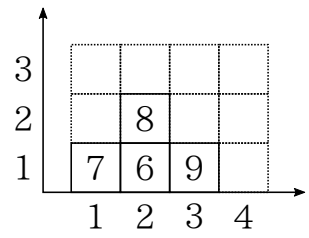

(g) $(5,2,4)$

Figure 7: An example of Theorem 1. (a)-(d): dominated sequence $(5,2,3),(7,1,4),(7,4,1)$, $(5,3,4),(\mathrm{e})-(\mathrm{g})$ : dominating sequence $(7,1,4),(7,4,1),(5,2,4)$

$\ldots,\left(i_{n-1}, s_{n-1}, d_{n-1}\right)$. Thus this sequence is admissible for $\mathscr{C}$. If the resulting configuration is denoted by $\widehat{\mathscr{C}}_{n-1}$, it satisfies $\widehat{\mathscr{C}}_{n-1} \leq L_{i_{1} d_{1} s_{1}}\left(\mathscr{C}_{n-1}\right)$. Therefore, from Lemmas 1 and 2 as well as (TA1), we obtain

$$
\begin{aligned}
R \circ L_{i_{1} s_{1} d_{n}}\left(\widehat{\mathscr{C}}_{n-1}\right) & \leq R \circ L_{i_{1} s_{1} d_{n}} \circ L_{i_{1} d_{1} s_{1}}\left(\mathscr{C}_{n-1}\right) \\
& =R \circ L_{i_{1} d_{1} d_{n}}\left(\mathscr{C}_{n-1}\right)=R \circ L_{i_{n} s_{n} d_{n}}\left(\mathscr{C}_{n-1}\right)=\mathscr{C}_{n} .
\end{aligned}
$$

This inequality implies that sequence $\left(i_{2}, s_{2}, d_{2}\right), \ldots,\left(i_{n-1}, s_{n-1}, d_{n-1}\right),\left(i_{1}, s_{1}, d_{n}\right)$ is admissible for $\mathscr{C}$ and the resulting configuration $R \circ L_{i_{1} s_{1} d_{n}}\left(\widehat{\mathscr{C}}_{n-1}\right)$ dominates $\mathscr{C}_{n}$.

It is easy to see that the above argument also holds true when $d_{n}=s_{1}$. In this case, sequence $\left(i_{2}, s_{2}, d_{2}\right), \ldots,\left(i_{n-1}, s_{n-1}, d_{n-1}\right)$ strictly dominates sequence $\left(i_{1}, s_{1}, d_{1}\right),\left(i_{2}, s_{2}, d_{2}\right), \ldots,\left(i_{n-1}, s_{n-1}, d_{n-1}\right),\left(i_{n}, s_{n}, d_{n}\right)$.

In the example of Figure 7 , sequence $(5,2,3),(7,1,4),(7,4,1),(5,3,4)$ satisfies the conditions in Theorem 1 , and is dominated by sequence $(7,1,4),(7,4,1)$, $(5,2,4)$. In this case, the dominating sequence is not feasible but admissible. Moreover, the resulting configuration is not identical to that by the dominated sequence.

Theorem 2. (Transitive Relocation Rule B) Sequence $\left(i_{1}, s_{1}, d_{n}\right),\left(i_{2}, s_{2}, d_{2}\right), \ldots$, $\left(i_{n-1}, s_{n-1}, d_{n-1}\right)$ strictly dominates sequence $\left(i_{1}, s_{1}, d_{1}\right),\left(i_{2}, s_{2}, d_{2}\right), \ldots,\left(i_{n}, s_{n}, d_{n}\right)$ if all the following conditions are satisfied: 
(TB1) $s_{n}=d_{1}, i_{n}=i_{1}$,

(TB2) $i_{1} \notin\left\{i_{2}, \ldots, i_{n-1}\right\}$,

(TB3) $d_{n} \notin\left\{s_{1}, d_{1}, \ldots, s_{n-1}, d_{n-1}\right\}$,

(TB4) $N_{d_{n}}\left(\mathscr{C}_{n-1}\right)=N_{d_{n}}(\mathscr{C})$.

Proof. From (TB4) and the feasibility of relocation $\left(i_{n}, s_{n}, d_{n}\right)$ for $\mathscr{C}_{n-1}, N_{d_{n}}(\mathscr{C})=$ $N_{d_{n}}\left(\mathscr{C}_{n-1}\right)<T$ holds. This inequality ensures the feasibility of relocation $\left(i_{1}, s_{1}, d_{n}\right)$ for $\mathscr{C}$, so that $\widehat{\mathscr{C}}_{1}$ is defined by $\widehat{\mathscr{C}}_{1}:=R \circ L_{i_{1} s_{1} d_{n}}(\mathscr{C})$. Because (TB3) and (TB4) imply that stack $d_{n}$ is not affected by sequence $\left(i_{1}, s_{1}, d_{1}\right), \ldots,\left(i_{n-1}, s_{n-1}, d_{n-1}\right)$ for $\mathscr{C}$, block $i_{1}$ does not interfere any retrieval caused by this sequence for $\mathscr{C}$, even if it is relocated to stack $d_{n}$ beforehand. Moreover, from (TB2), block $i_{1}$ is not relocated by sequence $\left(i_{2}, s_{2}, d_{2}\right), \ldots,\left(i_{n-1}, s_{n-1}, d_{n-1}\right)$. Therefore, this sequence is admissible for $\widehat{\mathscr{C}}_{1}$. If the resulting configuration is denoted by $\widehat{\mathscr{C}}_{n-1}$, it satisfies $\widehat{\mathscr{C}}_{n-1} \leq L_{i_{1} d_{1} d_{n}}\left(\mathscr{C}_{n-1}\right)$. By noting (TB1), we obtain

$$
\widehat{\mathscr{C}}_{n-1}=R\left(\widehat{\mathscr{C}}_{n-1}\right) \leq R \circ L_{i_{1} d_{1} d_{n}}\left(\mathscr{C}_{n-1}\right)=\mathscr{C}_{n} .
$$

This completes the proof.

An example of Theorem 2 is presented in Figure 8, where sequence $(6,2,3)$, $(8,1,2),(6,3,4)$ is dominated by sequence $(6,2,4),(8,1,2)$.

Theorem 3. (Transitive Relocation Rule C) Sequence $\left(i_{1}, s_{1}, d_{1}^{\prime}\right),\left(i_{2}, s_{2}, d_{2}\right), \ldots$, $\left(i_{n-1}, s_{n-1}, d_{n-1},\right)\left(i_{1}, d_{1}^{\prime}, d_{n}\right)$ dominates sequence $\left(i_{1}, s_{1}, d_{1}\right),\left(i_{2}, s_{2}, d_{2}\right), \ldots,\left(i_{n}, s_{n}, d_{n}\right)$ if all the following conditions are satisfied:

(TC1) $s_{n}=d_{1}, i_{n}=i_{1}$,

(TC2) $i_{1} \notin\left\{i_{2}, \ldots, i_{n-1}\right\}$,

(TC3) $d_{1}^{\prime} \notin\left\{s_{1}, d_{1}, s_{2}, d_{2}, \ldots, s_{n-1}, d_{n-1}, d_{n}\right\}$,

(TC4) $N_{d_{1}^{\prime}}\left(\mathscr{C}_{n-1}\right)=N_{d_{1}^{\prime}}(\mathscr{C})<T$.

Proof. From (TC4), relocation $\left(i_{1}, s_{1}, d_{1}^{\prime}\right)$ is feasible for $\mathscr{C}$. Thus let us define $\widehat{\mathscr{C}_{1}}:=R \circ L_{i_{1} s_{1} d_{1}^{\prime}}(\mathscr{C})$. Because (TC3) and (TC4) imply that stack $d_{1}^{\prime}$ is not affected by sequence $\left(i_{1}, s_{1}, d_{1}\right), \ldots,\left(i_{n-1}, s_{n-1}, d_{n-1}\right)$ for $\mathscr{C}$, block $i_{1}$ does not interfere any retrieval caused by this sequence for $\mathscr{C}$, even if it is relocated to 


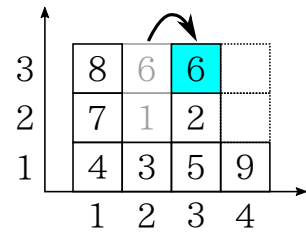

(a) $(6,2,3)$

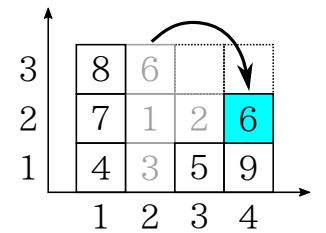

(d) $(6,2,4)$

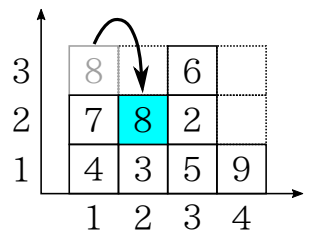

(b) $(8,1,2)$

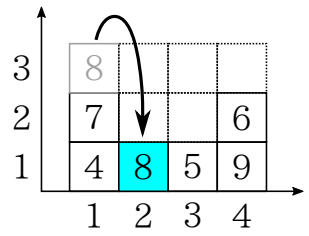

(e) $(8,1,2)$

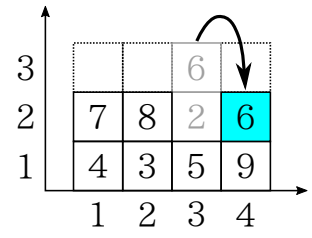

(c) $(6,3,4)$

Figure 8: An example of Theorem 2. (a)-(c): dominated sequence $(6,2,3),(8,1,2),(6,3,4)$, (d) and (e): dominating sequence $(6,2,4),(8,1,2)$

stack $d_{1}^{\prime}$ instead of stack $d_{1}$. Moreover, from (TC2), block $i_{1}$ is not relocated by sequence $\left(i_{2}, s_{2}, d_{2}\right), \ldots,\left(i_{n-1}, s_{n-1}, d_{n-1}\right)$. Therefore, this sequence is admissible for $\widehat{\mathscr{C}}_{1}$. If the resulting configuration is denoted by by $\widehat{\mathscr{C}}_{n-1}$, it satisfies $\widehat{\mathscr{C}}_{n-1} \leq L_{i_{1} d_{1} d_{1}^{\prime}}\left(\mathscr{C}_{n-1}\right)$. By noting (TC1), we obtain

$$
R \circ L_{i_{1} d_{1}^{\prime} d_{n}}\left(\widehat{\mathscr{C}}_{n-1}\right) \leq R \circ L_{i_{1} d_{1}^{\prime} d_{n}} \circ L_{i_{1} d_{1} d_{1}^{\prime}}\left(\mathscr{C}_{n-1}\right)=R \circ L_{i_{1} d_{1} d_{n}}\left(\mathscr{C}_{n-1}\right)=\mathscr{C}_{n} .
$$

It follows that sequence $\left(i_{1}, s_{1}, d_{1}^{\prime}\right),\left(i_{2}, s_{2}, d_{2}\right), \ldots,\left(i_{n-1}, s_{n-1}, d_{n-1}\right),\left(i_{1}, d_{1}^{\prime}, d_{n}\right)$ is admissible for $\mathscr{C}$, and the resulting configuration $R \circ L_{i_{1} d_{1}^{\prime} d_{n}}\left(\widehat{\mathscr{C}}_{n-1}\right)$ dominates $\mathscr{C}_{n}$.

In the example of Figure 9, sequence $(7,1,2),(4,1,4),(7,2,4)$ is dominated by sequence $(7,1,3),(4,1,4),(7,3,4)$.

\subsection{Dominance properties for independent relocations}

Next, two dominance properties on a pair of independent relocations are presented.

Theorem 4. (Independent Relocation Rule A) Sequence $\left(i_{2}, s_{2}, d_{2}\right), \ldots,\left(i_{n}, s_{n}, d_{n}\right)$, $\left(i_{1}, s_{1}, d_{1}\right)$ dominates sequence $\left(i_{1}, s_{1}, d_{1}\right),\left(i_{2}, s_{2}, d_{2}\right), \ldots,\left(i_{n}, s_{n}, d_{n}\right)$ if all the following conditions are satisfied:

(IA1) $\left\{s_{1}, d_{1}\right\} \cap\left\{s_{2}, d_{2}, \ldots, s_{n}, d_{n}\right\}=\emptyset$, 


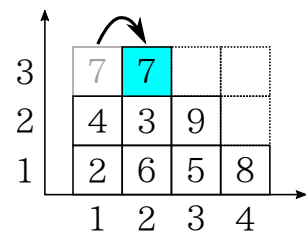

(a) $(7,1,2)$

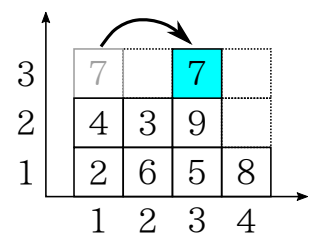

(d) $(7,1,3)$

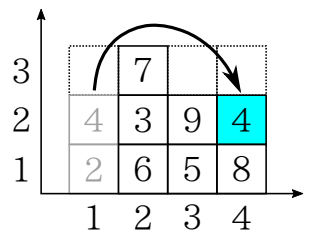

(b) $(4,1,4)$

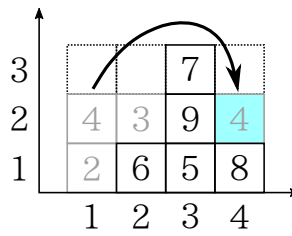

(e) $(4,1,4)$

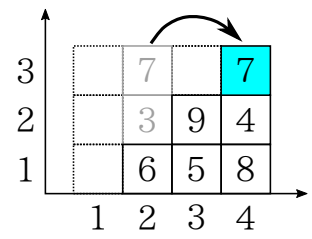

(c) $(7,2,4)$

Figure 9: An example of Theorem 3. (a)-(c): dominated sequence $(7,1,2),(4,1,4),(7,2,4)$, (d)-(f): dominating sequence $(7,1,3),(4,1,4),(7,3,4)$

(IA2) $N_{s_{1}}\left(\mathscr{C}_{n-1}\right)=N_{s_{1}}(\mathscr{C})-1$.

Proof. From (IA1) and (IA2), stack $s_{1}$ is not affected by sequence $\left(i_{1}, s_{1}, d_{1}\right), \ldots$, $\left(i_{n-1}, s_{n-1}, d_{n-1}\right)$ for $\mathscr{C}$ after block $i_{1}$ is relocated to stack $d_{1}$. The configuration of stack $s_{1}$ in $\mathscr{C}_{1}, \ldots, \mathscr{C}_{n-1}$ is the same as that in $\mathscr{C}$, except the absence of block $i_{1}$. Therefore, block $i_{1}$ does not interfere any retrieval caused by sequence $\left(i_{1}, s_{1}, d_{1}\right), \ldots,\left(i_{n-1}, s_{n-1}, d_{n-1}\right)$ for $\mathscr{C}$, even if it is not relocated to stack $d_{1}$. Hence $\left(i_{2}, s_{2}, d_{2}\right), \ldots,\left(i_{n}, s_{n}, d_{n}\right)$ is admissible for $\mathscr{C}$. Let us denote the resulting configuration by $\widehat{\mathscr{C}}_{n-1}$. If $i_{1} \notin \widehat{\mathscr{C}}_{n-1}$, it is obvious that relocation $\left(i_{1}, s_{1}, d_{1}\right)$ is admissible for $\widehat{\mathscr{C}}_{n-1}$, and $R \circ L_{i_{1} s_{1} d_{1}}\left(\widehat{\mathscr{C}}_{n-1}\right)=\widehat{\mathscr{C}}_{n-1} \leq \mathscr{C}_{n}$ holds. If $i_{1} \in \widehat{\mathscr{C}}_{n-1}$ and $i_{1} \in$ $\mathscr{C}_{n}$, block $i_{1}$ is on the top of stack $d_{1}$ in $\mathscr{C}_{n}$ and $N_{d_{1}}\left(\mathscr{C}_{n}\right)=N_{d_{1}}(\mathscr{C})+1 \leq T$ holds, so that relocation $\left(i_{1}, s_{1}, d_{1}\right)$ is feasible for $\widehat{\mathscr{C}}_{n-1}$ from $N_{d_{1}}\left(\widehat{\mathscr{C}}_{n-1}\right) \leq N_{d_{1}}(\mathscr{C})=$ $N_{d_{1}}\left(\mathscr{C}_{n}\right)-1<T$. Furthermore, $R \circ L_{i_{1} s_{1} d_{1}}\left(\widehat{\mathscr{C}}_{n-1}\right) \leq R \circ L_{i_{n} s_{n} d_{n}}\left(\mathscr{C}_{n-1}\right)=\mathscr{C}_{n}$ holds. If $i_{1} \in \widehat{\mathscr{C}}_{n-1}$ and $i_{1} \notin \mathscr{C}_{n}, i_{1} \in \mathscr{C}_{n-1}$ holds; otherwise, block $i_{1}$ should also be retrieved by sequence $\left(i_{2}, s_{2}, d_{2}\right), \ldots,\left(i_{n-1}, s_{n-1}, d_{n-1}\right)$ for $\mathscr{C}$ because block $i_{1}$ does not interfere any retrieval even if it is not relocated to stack $d_{1}$, which contradicts $i_{1} \in \widehat{\mathscr{C}}_{n-1}$. Because block $i_{1}$ is retrieved by the retrieval from $L_{i_{n} s_{n} d_{n}}\left(\mathscr{C}_{n-1}\right)$, relocation $\left(i_{1}, s_{1}, d_{1}\right)$ is feasible for $\widehat{\mathscr{C}}_{n-1}$ from $N_{d_{1}}\left(\widehat{\mathscr{C}}_{n-1}\right) \leq N_{d_{1}}(\mathscr{C})=N_{d_{1}}\left(\mathscr{C}_{n-1}\right)-1<$ $T$. Therefore, $R \circ L_{i_{1} s_{1} d_{1}}\left(\widehat{\mathscr{C}}_{n-1}\right) \leq R \circ L_{i_{n} s_{n} d_{n}}\left(\mathscr{C}_{n-1}\right)=\mathscr{C}_{n}$ holds also in this case. To summarize, $\left(i_{2}, s_{2}, d_{2}\right), \ldots,\left(i_{n}, s_{n}, d_{n}\right),\left(i_{1}, s_{1}, d_{1}\right)$ is admissible for $\mathscr{C}$ and the resulting configuration $R \circ L_{i_{1} s_{1} d_{1}}\left(\widehat{\mathscr{C}}_{n-1}\right)$ dominates $\mathscr{C}_{n}$. 


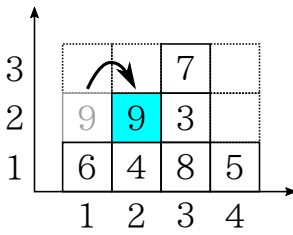

(a) $(9,1,2)$

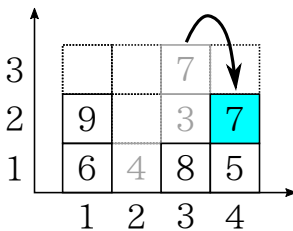

(c) $(7,3,4)$

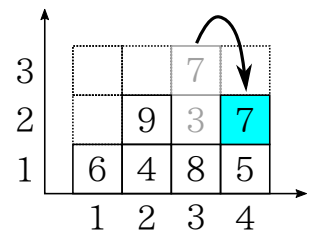

(b) $(7,3,4)$

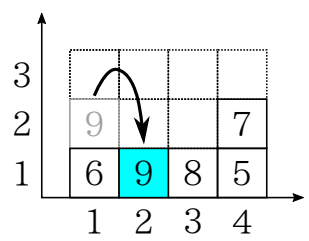

(d) $(9,1,2)$

Figure 10: An example of Theorems 4 and 5. (a) and (b): dominated sequence $(9,1,2),(7,3,4)$, (c) and (d): dominating sequence $(7,3,4),(9,1,2)$

Theorem 5. (Independent Relocation Rule B) Sequence $\left(i_{n}, s_{n}, d_{n}\right),\left(i_{1}, s_{1}, d_{1}\right)$, $\ldots,\left(i_{n-1}, s_{n-1}, d_{n-1}\right)$ dominates sequence $\left(i_{1}, s_{1}, d_{1}\right),\left(i_{2}, s_{2}, d_{2}\right), \ldots,\left(i_{n}, s_{n}, d_{n}\right)$ if all the following conditions are satisfied:

(IB1) $\left\{s_{n}, d_{n}\right\} \cap\left\{s_{1}, d_{1}, \ldots, s_{n-1}, d_{n-1}\right\}=\emptyset$,

(IB2) $N_{s_{n}}\left(\mathscr{C}_{n-1}\right)=N_{s_{n}}(\mathscr{C})$,

(IB3) $N_{d_{n}}\left(\mathscr{C}_{n-1}\right)=N_{d_{n}}(\mathscr{C})$.

Proof. From (IB1), (IB2) and (IB3), stacks $s_{n}$ and $d_{n}$ are unaffected by sequence $\left(i_{1}, s_{1}, d_{1}\right), \ldots,\left(i_{n-1}, s_{n-1}, d_{n-1}\right)$ for $\mathscr{C}$. Therefore, sequence $\left(i_{n}, s_{n}, d_{n}\right),\left(i_{1}, s_{1}, d_{1}\right)$, $\ldots,\left(i_{n-1}, s_{n-1}, d_{n-1}\right)$ is admissible for $\mathscr{C}$ from the feasibility of relocation $\left(i_{n}, s_{n}, d_{n}\right)$ for $\mathscr{C}_{n-1}$. If the resulting configuration is denoted by $\widehat{\mathscr{C}}_{n}, \widehat{\mathscr{C}}_{n} \leq L_{i_{n} s_{n} d_{n}}\left(\mathscr{C}_{n-1}\right)$ holds, and we obtain

$$
\widehat{\mathscr{C}}_{n}=R\left(\widehat{\mathscr{C}}_{n}\right) \leq R \circ L_{i_{n} s_{n} d_{n}}\left(\mathscr{C}_{n-1}\right)=\mathscr{C}_{n}
$$

This completes the proof.

Figure 10 illustrates an example of Theorems 4 and 5. Both the theorems are applicable and sequence $(9,1,2),(7,3,4)$ is proved to be dominated by sequence $(7,3,4),(9,1,2)$. 


\subsection{Dominance properties for retrieval}

Finally, two dominance properties on retrieval are proved.

Theorem 6. (Retrieval Rule A) Sequence $\left(i_{2}, s_{2}, d_{2}\right), \ldots,\left(i_{n}, s_{n}, d_{n}\right)$ strictly dominates sequence $\left(i_{1}, s_{1}, d_{1}\right),\left(i_{2}, s_{2}, d_{2}\right), \ldots,\left(i_{n}, s_{n}, d_{n}\right)$ if all the following conditions are satisfied:

(RA1) $i_{1} \notin\left\{i_{2}, \ldots, i_{n-1}, i_{n}\right\}$,

(RA2) $i_{1} \in \mathscr{C}_{n-1} \wedge i_{1} \notin \mathscr{C}_{n}$,

(RA3) $s_{1} \notin\left\{s_{2}, d_{2}, \ldots, s_{n}, d_{n}\right\}$,

(RA4) $\left|\mathscr{V}_{s_{1}}(\mathscr{C})\right|=\left|\mathscr{V}_{s_{1}}\left(\mathscr{C}_{n-1}\right)\right|$.

Proof. From (RA4), the priority of block $i_{1}$ is higher (smaller) than the other blocks in stack $s_{1}$ in $\mathscr{C}$. Thus these blocks should appear in $L_{i_{n} s_{n} d_{n}}\left(\mathscr{C}_{n-1}\right)$, because (RA2) implies that block $i_{1}$, which should be retrieved before them, is retrieved by the retrieval from $L_{i_{n} s_{n} d_{n}}\left(\mathscr{C}_{n-1}\right)$. Furthermore, from (RA3), no block is relocated from or to stack $s_{1}$ by sequence $\left(i_{2}, s_{2}, d_{2}\right), \ldots,\left(i_{n}, s_{n}, d_{n}\right)$. Therefore, the block configuration of stack $s_{1}$ in $\mathscr{C}_{1}, \ldots, \mathscr{C}_{n-1}$, and $L_{i_{n} s_{n} d_{n}}\left(\mathscr{C}_{n-1}\right)$ is the same as that in $\mathscr{C}$ except the absence of block $i_{1}$. It follows that block $i_{1}$ does not interfere any retrieval caused by sequence $\left(i_{1}, s_{1}, d_{1}\right), \ldots,\left(i_{n}, s_{n}, d_{n}\right)$ for $\mathscr{C}$ even if it is not relocated to stack $d_{1}$, except the retrieval from $L_{i_{n} s_{n} d_{n}}\left(\mathscr{C}_{n-1}\right)$. Because (RA1) ensures that block $i_{1}$ is not relocated by sequence $\left(i_{2}, s_{2}, d_{2}\right), \ldots,\left(i_{n}, s_{n}, d_{n}\right)$, this sequence is admissible for $\mathscr{C}$. By further noting that no block is placed on block $i_{1}$ by this sequence for $\mathscr{C}$, we can see that block $i_{1}$ is retrieved also in the resulting configuration, so that it dominates $\mathscr{C}_{n}$.

Figure 11 provides an example of Theorem 11 where sequence $(4,1,2),(7,4,3)$ is dominated by sequence $(7,4,3)$.

Theorem 7. (Retrieval Rule B) Sequence $\left(i_{1}, s_{1}, d_{1}^{\prime}\right),\left(i_{2}, s_{2}, d_{2}\right), \ldots,\left(i_{n}, s_{n}, d_{n}\right)$ dominates sequence $\left(i_{1}, s_{1}, d_{1}\right),\left(i_{2}, s_{2}, d_{2}\right), \ldots,\left(i_{n}, s_{n}, d_{n}\right)$ if all the following conditions are satisfied:

(RB1) $i_{1} \notin\left\{i_{2}, \ldots, i_{n-1}, i_{n}\right\}$,

(RB2) $i_{1} \in \mathscr{C}_{n-1} \wedge i_{1} \notin \mathscr{C}_{n}$,

(RB3) $d_{1}^{\prime} \notin\left\{s_{1}, d_{1}, \ldots, s_{n}, d_{n}\right\}$, 


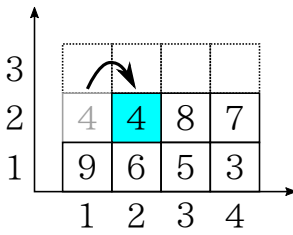

(a) $(4,1,2)$

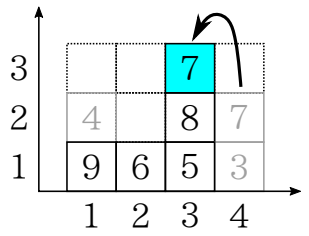

(c) $(7,4,3)$

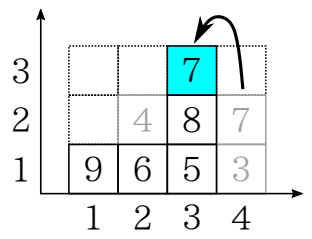

(b) $(7,4,3)$

Figure 11: An example of Theorem 6. (a) and (b): dominated sequence $(4,1,2),(7,4,3)$, (c): dominating sequence $(7,4,3)$

(RB4) $N_{d_{1}^{\prime}}(\mathscr{C})<T$,

(RB5) $Q_{d_{1}^{\prime}}(\mathscr{C})>i_{1}$.

Proof. From (RB5), blocks in stack $d_{1}^{\prime}$ in $\mathscr{C}$ are not retrieved before block $i_{1}$ that, from (RB2), should be retrieved by the retrieval for $L_{i_{n} s_{n} d_{n}}\left(\mathscr{C}_{n-1}\right)$. Thus these blocks should appear in $L_{i_{n} s_{n} d_{n}}\left(\mathscr{C}_{n-1}\right)$. Furthermore, from (RB3), no block is relocated from or to stack $d_{1}^{\prime}$ by sequence $\left(i_{1}, s_{1}, d_{1}\right), \ldots,\left(i_{n}, s_{n}, d_{n}\right)$ for $\mathscr{C}$. It follows that the block configuration of stack $d_{1}^{\prime}$ in $\mathscr{C}, \mathscr{C}_{1}, \ldots, \mathscr{C}_{n-1}$, and $L_{i_{n} s_{n} d_{n}}\left(\mathscr{C}_{n-1}\right)$ is the same. This fact together with the feasibility of relocation $\left(i_{1}, s_{1}, d_{1}^{\prime}\right)$ for $\mathscr{C}$ from (RB4) ensures that block $i_{1}$ does not interfere any retrieval caused by sequence $\left(i_{1}, s_{1}, d_{1}\right), \ldots,\left(i_{n}, s_{n}, d_{n}\right)$ for $\mathscr{C}$ even if it is relocated to stack $d_{1}^{\prime}$ instead of stack $d_{1}$, except the retrieval from $L_{i_{n} s_{n} d_{n}}\left(\mathscr{C}_{n-1}\right)$. Therefore, sequence $\left(i_{1}, s_{1}, d_{1}^{\prime}\right)$, $\left(i_{2}, s_{2}, d_{2}\right), \ldots,\left(i_{n}, s_{n}, d_{n}\right)$ is admissible for $\mathscr{C}$. By further noting that no block is placed on block $i_{1}$ by this sequence for $\mathscr{C}$, we can see that block $i_{1}$ is retrieved also in the resulting configuration, so that it dominates $\mathscr{C}_{n}$.

In Figure 12, sequence $(4,1,2),(7,4,1)$ dominates sequence $(4,1,3),(7,4,1)$ and vice versa according to Theorem 7 . Therefore, not both of the sequences should be forbidden in the branch-and-bound algorithm as noted in 3.1.

\subsection{Dominance properties for the restricted BRP}

Although Theorems 1-7 are originally meant for the unrestricted BRP, some of them are applicable also to the restricted BRP. However, it should be noted that 


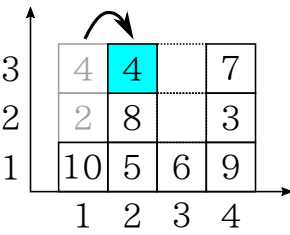

(a) $(4,1,2)$

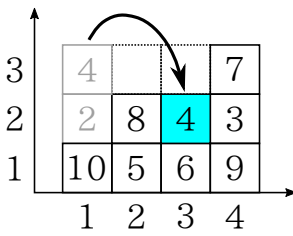

(c) $(4,1,3)$

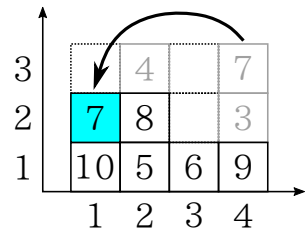

(b) $(7,4,1)$

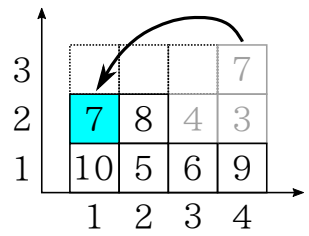

(d) $(7,4,1)$

Figure 12: An example of Theorem 7. (a) and (b): sequence $(4,1,2),(7,4,1)$, (c) and (d): sequence $(4,1,3),(7,4,1)$. The two sequences dominate each other

source stacks of relocations are uniquely determined in the restricted BRP. For example, in Theorem 1 , sequence $\left(i_{2}, s_{2}, d_{2}\right), \ldots,\left(i_{n-1}, s_{n-1}, d_{n-1}\right),\left(i_{1}, s_{1}, d_{n}\right)$ is admissible for $\mathscr{C}$ in the case of the unrestricted BRP. In order for this sequence to be admissible for the restricted BRP, the target block should be in stack $s_{1}$ when relocation $\left(i_{1}, s_{1}, d_{n}\right)$ is performed. However, it is not the case in general and the sequence can be infeasible. In view of this, only Theorems 2 and 7 are applicable to the restricted BRP. Theorem 2 holds without any modification, whereas (RB4) should be replaced by the following condition in Theorem 7:

(RB4') $N_{d_{1}^{\prime}}(\mathscr{C}) \leq N_{d_{1}}(\mathscr{C})$.

In (RB2) of Theorem 7, block $i_{1}$ in stack $d_{1}$ is assumed to be retrieved from $L_{i_{n} s_{n} d_{n}}\left(\mathscr{C}_{n-1}\right)$. It follows that block $i_{1}$ becomes the target block at some point, and only blocks above it are relocatable after that in the restricted BRP. To consider an admissible dominating sequence, all the blocks relocated to (from) stack $d_{1}$ after block $i_{1}$ in sequence $\left(i_{1}, s_{1}, d_{1}\right),\left(i_{2}, s_{2}, d_{2}\right), \ldots,\left(i_{n}, s_{n}, d_{n}\right)$ should also be relocated to (resp. from) stack $d_{1}^{\prime}$, Therefore, we should consider sequence $\left(i_{1}, s_{1}, d_{1}^{\prime}\right),\left(i_{2}, s_{2}^{\prime}, d_{2}^{\prime}\right), \ldots,\left(i_{n}, s_{n}^{\prime}, d_{n}^{\prime}\right)$ instead of sequence $\left(i_{1}, s_{1}, d_{1}^{\prime}\right),\left(i_{2}, s_{2}, d_{2}\right)$, $\ldots,\left(i_{n}, s_{n}, d_{n}\right)$, where, for $k=2, \ldots, n$.

$$
s_{k}^{\prime}=\left\{\begin{array}{ll}
d_{1}^{\prime}, & s_{k}=d_{1}, \\
s_{k}, & s_{k} \neq d_{1},
\end{array} \quad d_{k}^{\prime}= \begin{cases}d_{1}^{\prime}, & d_{k}=d_{1}, \\
d_{k}, & d_{k} \neq d_{1} .\end{cases}\right.
$$




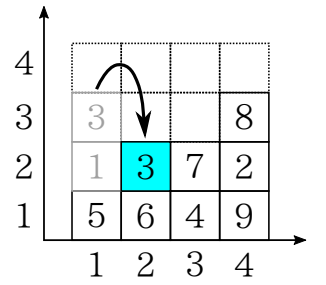

(a) $(3,1,2)$

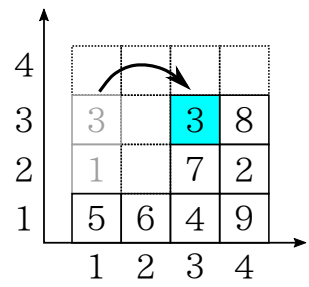

(d) $(3,1,3)$

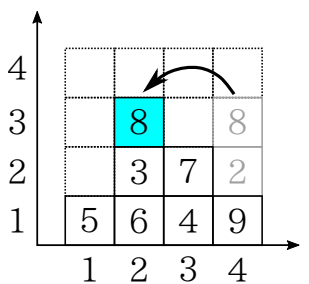

(b) $(8,4,2)$

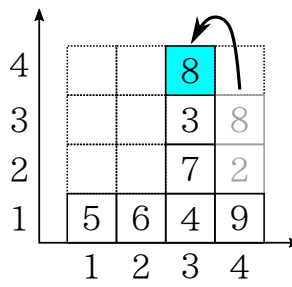

(e) $(8,4,3)$

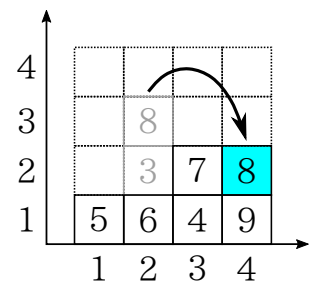

(c) $(8,2,4)$

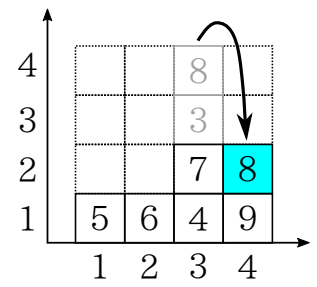

(f) $(8,3,4)$

Figure 13: An example of Theorem 7 for the restricted BRP. (a)-(c): sequence $(3,1,2),(8,4,2)$, $(8,2,4)$, (d)-(f): sequence $(3,1,3),(8,4,3),(8,3,4)$. The two sequences dominate each other

To make this sequence admissible, stack $d_{1}^{\prime}$ should be as slack as stack $d_{1}$, which is ensured by $N_{d_{1}^{\prime}}(\mathscr{C}) \leq N_{d_{1}}(\mathscr{C})$.

In the example of Figure 13, two sequences dominate each other as in Figure 12. Please note that the relocations of block 8 are changed according to the change in the destination stack of block 3 .

\section{Consistency of dominance properties}

The theorems in the preceding section enable us to eliminate unnecessary nodes in the search tree of the branch-and-bound algorithm. However, they should be employed carefully so as not to exclude all optimal sequences. In the example of Figure 6, sequence $(3,2,1)$ dominates sequence $(3,2,3)$ and vice versa according to Theorem 7 with $n=1$, meaning that it is not sufficient to simply forbid dominated sequences. Two sequences dominate each other also in Figures 12 and 13. In this section we explain how to break such ties in the conditions to ensure the consistency of the dominance properties.

Theorems 1, 2, and 6 (Transitive Relocation Rules A and B, Retrieval Rule A) give strong dominance properties in the sense that they ensure the existence of a strictly dominating sequence with fewer relocations. On the other hand, the rest of the theorems only give weak dominance properties, and we should break ties 
consistently so as not to forbid all optimal sequences. The consistency is ensured based on stack priorities. In Theorem 3, ties are broken by adding the following condition:

(TC5) $d_{1} \in\left\{s_{2}, d_{2}, \ldots, s_{n-1}, d_{n-1}\right\} \vee Q_{d_{1}^{\prime}}(\mathscr{C})>Q_{d_{1}}(\mathscr{C})$.

Condition $d_{1} \in\left\{s_{2}, d_{2}, \ldots, s_{n-1}, d_{n-1}\right\}$ in (TC5) together with (TC3) means that we prefer a stack $d_{1}^{\prime}$ such that no block is relocated from or to stack $d_{1}^{\prime}$ by sequence $\left(i_{2}, s_{2}, d_{2}\right), \ldots,\left(i_{n}, s_{n}, d_{n}\right)$. If no block is relocated from or to stack $d_{1}$, either, a stack $d_{1}^{\prime}$ with the lower (larger) priority is preferred $\left(Q_{d_{1}^{\prime}}(\mathscr{C})>Q_{d_{1}}(\mathscr{C})\right)$. If a preferred stack $d_{1}^{\prime}$ exists, sequence $\left(i_{1}, s_{1}, d_{1}\right), \ldots,\left(i_{n}, s_{n}, d_{n}\right)$ is forbidden. The same applies to Theorem 7, and

(RB5') $\left(d_{1} \in\left\{s_{2}, d_{2}, \ldots, s_{n}, d_{n}\right\} \wedge Q_{d_{1}^{\prime}}(\mathscr{C})>i_{1}\right) \vee Q_{d_{1}^{\prime}}(\mathscr{C})>Q_{d_{1}}(\mathscr{C})$

is checked instead of (RB5). Condition $Q_{d_{1}^{\prime}}(\mathscr{C})>Q_{d_{1}}(\mathscr{C})$ in (RB5') implies (RB5) because from (RB1) and (RB2), block $i_{1}$ is retrieved before any block in stack $d_{1}$ of $\mathscr{C}$ by sequence $\left(i_{1}, s_{1}, d_{1}\right), \ldots,\left(i_{n}, s_{n}, d_{n}\right)$, so that $i_{1}<Q_{d_{1}}(\mathscr{C})<$ $Q_{d_{1}^{\prime}}(\mathscr{C})$ should hold.

Ties in Theorems 4 and 5 can be broken by adding the following conditions, respectively:

(IA3) $Q_{s_{1}}(\mathscr{C})>Q_{s_{n}}\left(\mathscr{C}_{n-1}\right)$.

(IB4) $Q_{s_{1}}(\mathscr{C})>Q_{s_{n}}\left(\mathscr{C}_{n-1}\right)$.

However, even if ties are broken appropriately in this manner, Theorems 4 and 5 are possibly inconsistent with each other. Figure 14 illustrates an example. In this case, $Q_{1}=3>Q_{4}=2$ and sequence $(8,1,2),(10,3,5),(5,4,5)$ satisfies (IA1)(IA3). Hence it is dominated by sequence $(10,3,5),(5,4,5),(8,1,2)$, and the former sequence is eliminated. However, $Q_{3}=4>Q_{4}=2$ and the latter satisfies (IB1)-(IB4), so that it is also eliminated. To avoid this kind of inconsistency, only either Theorem 4 or Theorem 5 should be used. According to results of computational experiments, there was no significant difference in the performance of the two theorems. Thus Theorem 4, which is a little simpler than Theorem 5, was used in our proposed algorithm.

To break ties in Theorem 7 for the restricted BRP, (RB4') and (RB5') are combined into

(RB4") $N_{d_{1}^{\prime}}(\mathscr{C})<N_{d_{1}}(\mathscr{C}) \vee\left(N_{d_{1}^{\prime}}(\mathscr{C})=N_{d_{1}}(\mathscr{C}) \wedge Q_{d_{1}}(\mathscr{C})<Q_{d_{1}^{\prime}}(\mathscr{C})\right)$.

In (RB4"), ties are broken by the stack height first, and next the stack priority. 


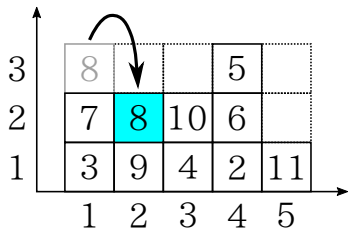

(a) $(8,1,2)$

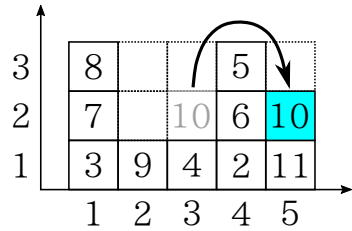

(d) $(10,3,5)$

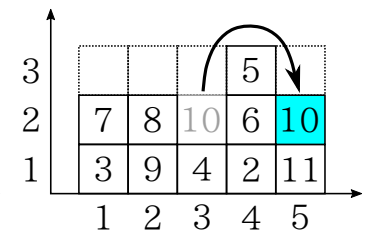

(b) $(10,3,5)$

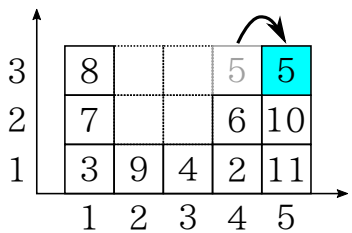

(e) $(5,4,5)$

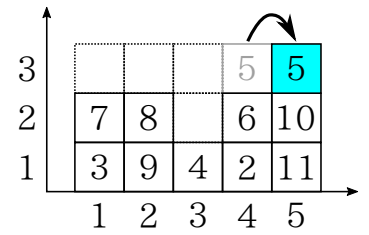

(c) $(5,4,5)$

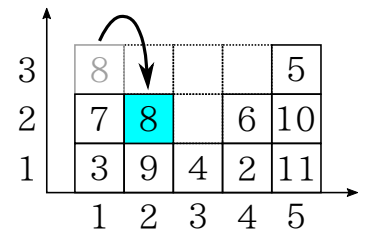

(f) $(8,1,2)$

Figure 14: An example of sequences for which (IA1)-(IA3) and (IB1)-(IB4) become inconsistent. (a)-(c): sequence $(8,1,2),(10,3,5),(5,4,5),(d)-(f)$ : sequence $(10,3,5),(5,4,5),(8,1,2)$. The former sequence is eliminated and the latter is adopted according to (IA1)-(IA3), whereas the former is adopted and the latter is eliminated according to (IB1)-(IB4)

\section{New lower bound}

One of the primary contributions of this study is the dominance properties in Section 4. The other is a new lower bound of the total number of relocations. It is an extension of the lower bound by Forster and Bortfeldt [12]. Kim and Hong [5] utilized the total number of blocking blocks as a lower bound in their branchand-bound algorithm because a blocking block should be relocated at least once in order to retrieve the block with a higher priority under it. Forster and Bortfeldt [12] showed that it can be increased by one if a BG relocation does not exist for any topmost blocking block. In this section we further improve it by relaxing the condition for the increment. In the following, a minimal configuration is assumed, and the lower bound by Kim and Hong [5] (the total number of blocking blocks) and that by Forster and Bortfeldt [12] are denoted by LB-KH and LB-FB, respectively.

\subsection{LB-FB: Lower bound by Forster and Bortfeldt [12]}

As a preparation, we investigate LB-FB in detail. If $\mathrm{LB}-\mathrm{KH}$ is equal to the optimal value, every relocation in an optimal sequence should decrease blocking blocks by one. In other words, an optimal sequence should be composed only of $\mathrm{BG}$ relocations. Because only topmost blocks are relocatable at first, the first relocation in the sequence is a $\mathrm{BG}$ relocation of a topmost blocking block. It 


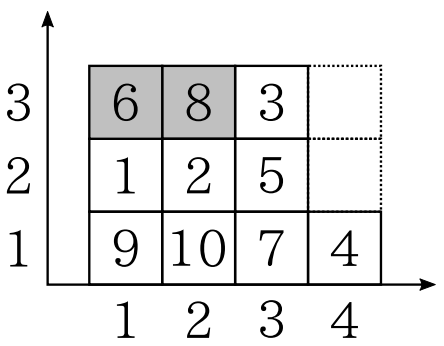

Figure 15: A configuration for which LB-FB succeeds in improving LB-KH. LB-KH $=2$, $\mathrm{LB}-\mathrm{FB}=3$, and gray blocks are blocking blocks

follows that the optimal value should be greater than $\mathrm{LB}-\mathrm{KH}$ if a $\mathrm{BG}$ relocation does not exist for any topmost blocking block. In this case, $\mathrm{LB}-\mathrm{KH}+1$ is a valid lower bound and LB-FB is given by LB-KH +1 . Otherwise, LB-FB is equal to LB-KH. In summary, LB-KH is increased by one if

$$
\min _{\substack{s \in \mathscr{S} \\ Q_{s}<P_{s}^{\text {top }}}} P_{s}^{\text {top }}>\max _{s \in \mathscr{S}} Q_{s}
$$

is satisfied. The lefthand side of (7) denotes the highest (smallest) priority of the topmost blocking blocks. In the configuration in Figure 15, the total number of blocking blocks (gray blocks) is 2 and hence LB-KH $=2$, whereas LB-FB $=$ $\mathrm{LB}-\mathrm{KH}+1=3$ because the highest (smallest) priority of the topmost blocking blocks is 6 and the lowest (largest) stack priority is 4 .

\subsection{Proposed lower bound $L B-N$}

In the proposed lower bound, a similar idea to LB-FB is exploited to check whether a BG relocation exists or not. The difference from LB-FB is that blocking blocks above the target block are checked. We first identify candidates for their destination stacks. Suppose that the target block is in the $t^{\mathrm{T}}$-th tier of stack $s^{\mathrm{T}}$. That is, the target block is block $P_{s^{\mathrm{T}}} t^{\mathrm{T}}$. Then, any stack $j \in \mathscr{S}^{\text {slack }} \backslash\left\{s^{\mathrm{T}}\right\}$ is obviously a candidate destination stack. Even if $N_{j}=T$, stack $j$ can be a candidate, provided that its topmost block is a blocking block and there exists a BG relocation for it. Therefore, the set of indices of candidate destination stacks is given by

$$
\mathscr{D}=\left\{j \in \mathscr{S} \backslash\left\{s^{\mathrm{T}}\right\} \mid N_{j}<T \vee Q_{j}<P_{j}^{\mathrm{top}}<\max _{s \in \mathscr{S}_{\text {slack }}} Q_{s}\right\}
$$




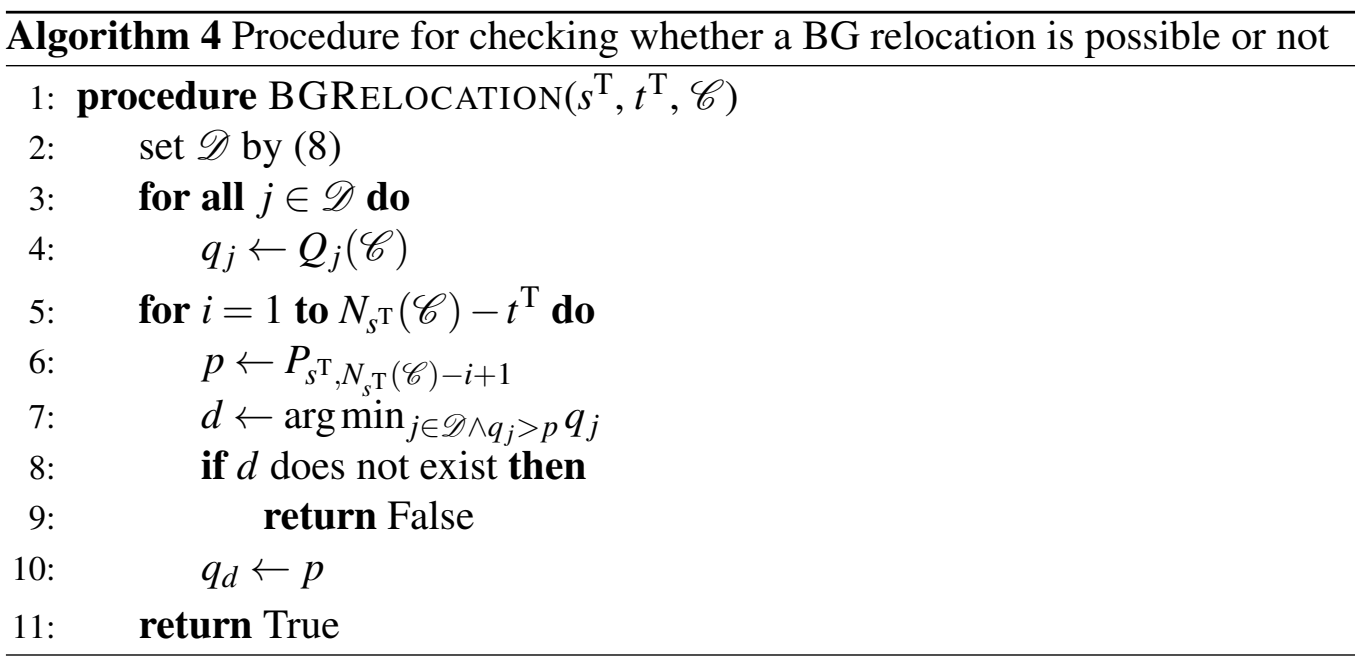

In (8), $Q_{j}<P_{j}^{\text {top }}$ means that the topmost block of stack $j$ is a blocking block, and $P_{j}^{\text {top }}<\max _{s \in \mathscr{S} \text { slack }} Q_{s}$ ensures that a BG relocation exists for this block. One may think that $\max _{s \in \mathscr{S} \text { slack }} Q_{s}$ should be replaced by $\max _{s \in \mathscr{S}} Q_{s}$ because the topmost blocking block of stack $j$ can be relocated to stack $s^{\prime}$ even when $N_{s^{\prime}}=T$, if the topmost block of stack $s^{\prime}$ is relocated beforehand. Suppose that such a stack $s^{\prime}$ satisfying $Q_{s^{\prime}}>\max _{s \in \mathscr{S} \text { slack }} Q_{s}$ exists. Because the topmost block of stack $s^{\prime}$ should also be relocated by a BG relocation, its priority $P_{s^{\prime}}^{\text {top }}$ satisfies $Q_{s^{\prime \prime}}>P_{s^{\prime}}^{\text {top }}>Q_{s^{\prime}}$ where stack $s^{\prime \prime}$ is the destination stack of this BG relocation. However, $N_{s^{\prime \prime}}=T$ holds from $Q_{s^{\prime \prime}}>Q_{s^{\prime}}>\max _{s \in \mathscr{S} \text { slack }} Q_{s}$, and hence a BG relocation should exist for the topmost blocking block $P_{s^{\prime \prime}}^{\text {top }}$ of stack $s^{\prime \prime}$ to relocate it beforehand. By repeatedly applying this argument, we can see that no BG relocation exists for the topmost blocking block of stack $j$. Thus stacks $j$ with $P_{j}^{\text {top }}>Q_{j}>\max _{s \in \mathscr{S} \text { slack }} Q_{s}$ are excluded from the candidate destination stacks.

Next, let us consider the priorities of the candidate destination stacks. We should take into account the effect of relocating blocks other than those above the target block. Because these relocations are BG relocations, they do not affect the priorities of their source stacks, while they always make the priorities of their destination stacks higher (smaller). It follows that the current priorities of the candidate destination stacks yield lowest possible values (upper bounds) of their actual priorities. Since our purpose here is to obtain a lower bound of the total number of relocations, we can safely assume that the priorities of the candidate destination stacks $j \in \mathscr{D}$ are given by their current values $Q_{j}$.

Now, let us check whether a BG relocation exists or not for the blocks above 


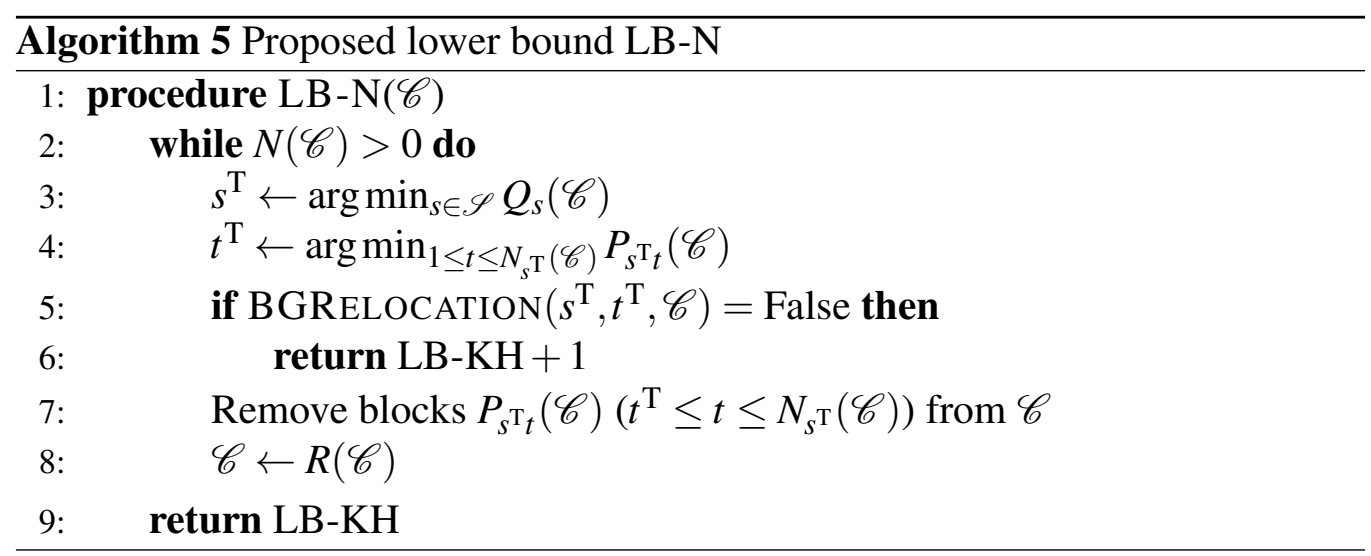

the target block, blocks $P_{s^{\mathrm{T}} N_{s}^{\mathrm{T}}}, P_{s^{\mathrm{T}}, N_{s}-1}, \ldots, P_{s^{\mathrm{T}}, t^{\mathrm{T}}+1}$ in this order. According to Lemma 1 in Tanaka and Takii [23], it can be checked easily if the height limit is ignored. Specifically, a BG relocation exists for any block above the target block if Algorithm 4 is feasible. If it is infeasible, that is, $d$ is not found on line 7, a BG relocation does not exist for some block. In this case, LB-KH can be increased by one. The time complexity of this algorithm is given by $O\left(\left(N_{s^{\mathrm{T}}}-t^{\mathrm{T}}\right) \log S\right)$ if the candidate destination stacks are sorted in the increasing order of their priorities beforehand. It is because this order is kept unchanged even if $q_{d}$ is updated on line 10, and $d$ can always be found in $O(\log S)$ time on line 7.

We apply this check in the framework of LB3 by Zhu et al. [13]. If no BG relocation exists for some block above the target block, LB-KH is increased by one. Otherwise, the blocks above the target block are removed from the current configuration together with the target block. Then, the same check is applied to the blocks above the new target block. This procedure is repeated until it is proved that LB-KH can be increased by one, or all the blocks are removed. The algorithm for computing the proposed lower bound LB-N is presented in Algorithm 5.

The validity of LB-N is verified as follows. Let us consider a minimal configuration $\mathscr{C}$ and denote the target block in $\mathscr{C}$ by block $x$. Let us also denote by $\mathscr{C}^{\prime}$, the configuration obtained by removing the blocks above block $x$ as well as block $x$ itself, and by block $x^{\prime}$, the target block in $\mathscr{C}^{\prime}$. Then, it suffices to show that if $f^{*}(\mathscr{C})=\mathrm{LB}-\mathrm{KH}$, a BG relocation exists for any block above block $x^{\prime}$ in $\mathscr{C}^{\prime}$ because the contraposition holds. From $f^{*}(\mathscr{C})=\mathrm{LB}-\mathrm{KH}$, all relocations in an optimal sequence should be BG relocations, so that all blocking blocks in $\mathscr{C}$ including those above block $x$ are relocated by BG relocations. Because BG relocations for these blocks always make the priorities of their destination stacks 

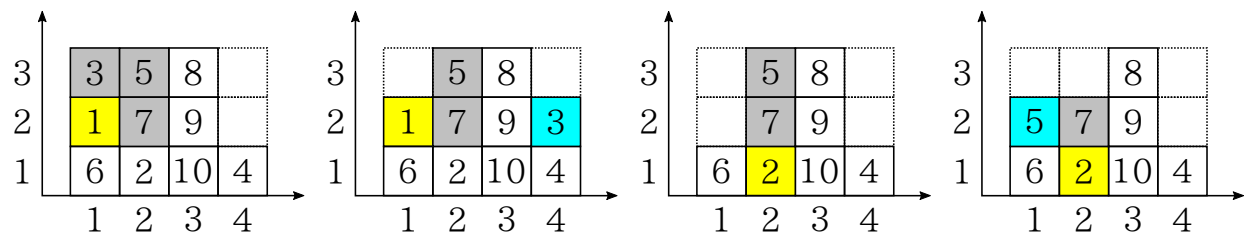

(a) Initial configura- (b) Relocation of (c) Blocks 3 and 1 (d) Relocation of tion. Block 1 is the block 3 to stack 4 is are removed. Block block 5 to stack 1 is target block. $\mathscr{D}=$ a BG relocation $\{4\}$ 2 is the next target a BG relocation block. $\mathscr{D}=\{1,4\}$

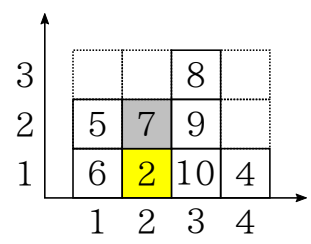

(e) No BG relocation exists for block 7

Figure 16: An example of calculation of LB-N for a configuration for which $\mathrm{LB}-\mathrm{N}=4$ is greater than $\mathrm{LB}-\mathrm{FB}(=\mathrm{LB}-\mathrm{KH}=3)$

higher (smaller), the actual priorities of the destination stacks of blocks above block $x^{\prime}$ in $\mathscr{C}$ are not lower (larger) than than those in $\mathscr{C}^{\prime}$. It follows that a BG relocation should exist for any block above block $x^{\prime}$ also in $\mathscr{C}^{\prime}$.

It is not difficult to show that LB-N always dominates LB-FB. If (7) is satisfied,

$$
P_{s^{\mathrm{T}}}^{\text {top }} \geq \min _{\substack{s \in \mathscr{S} \\ Q_{s}<P_{s}^{\text {top }}}} P_{s}^{\text {top }}>\max _{s \in \mathscr{S}} Q_{s} \geq \max _{s \in \mathscr{D}} Q_{s}
$$

holds. It follows that the procedure in Algorithm 4 is infeasible for the topmost block $P_{s^{\mathrm{T}}}^{\text {top }}=P_{s^{\mathrm{T}} N_{s} \mathrm{~T}}$ of stack $s^{\mathrm{T}}$, so that LB-N $=\mathrm{LB}-\mathrm{KH}+1$.

Figure 16 illustrates the procedure of LB-N. We note that LB-FB $=\mathrm{LB}-\mathrm{KH}=$ 3 for the configuration in (a). First, we check block 3 above the target block 1 . The set of indices of candidate destination stacks in (8) is $\mathscr{D}=\{4\}$ because no $\mathrm{BG}$ relocation exists for the topmost blocking block 5 of stack 2 , and the topmost block 8 of stack 3 is not a blocking block. Because the relocation of block 3 to stack $4(\in \mathscr{D})$ is a BG relocation (b), we remove this block and then the target block. Now, block 2 becomes the target block and $\mathscr{D}=\{1,4\}$ (c). Thus we check block 5 , and it can be relocated to stack 1 by a BG relocation (d). We next check 
block 7, for which no BG relocation is possible (e). Finally, it is proved that $\mathrm{LB}-\mathrm{KH}$ can be increased by one and LB-N is given by LB-KH $+1=4$.

\subsection{Speed-up of lower bound computation}

A lower bound is computed at every node in the search tree of the branch-andbound algorithm. Compared to LB-KH that can be computed incrementally in $O(1)$ time from that for the parent node, LB-N needs a longer computation time. Here, we show that we need not compute LB-N at some nodes, where we can obtain a lower bound in $O(1)$ time.

Let us denote $\mathrm{LB}-\mathrm{KH}$ and $\mathrm{LB}-\mathrm{N}$ for $\mathscr{C}$ by $\mathrm{LB}-\mathrm{KH}(\mathscr{C})$ and $\mathrm{LB}-\mathrm{N}(\mathscr{C})$, respectively. Let us also denote by $\mathscr{C}^{\text {parent }}$ and $\mathscr{C}^{\text {cur }}$ the configurations represented by the parent and current nodes, respectively. Because $\mathscr{C}^{\text {cur }}$ is obtained by relocating one block in $\mathscr{C}^{\text {parent }}, f^{*}\left(\mathscr{C}^{\text {cur }}\right)+1 \geq \mathrm{LB}-\mathrm{N}\left(\mathscr{C}^{\text {parent }}\right)$ should hold due to the validity of LB-N $\left(\mathscr{C}^{\text {parent }}\right)$ as a lower bound. Therefore, if both $\mathrm{LB}-\mathrm{N}\left(\mathscr{C}^{\text {parent }}\right)=$ $\mathrm{LB}-\mathrm{KH}\left(\mathscr{C}^{\text {parent }}\right)+1$ and $\mathrm{LB}-\mathrm{KH}\left(\mathscr{C}^{\text {cur }}\right)=\mathrm{LB}-\mathrm{KH}\left(\mathscr{C}^{\text {parent }}\right)-1$ hold, we obtain

$$
f^{*}\left(\mathscr{C}^{\text {cur }}\right) \geq \mathrm{LB}-\mathrm{N}\left(\mathscr{C}^{\text {parent }}\right)-1=\mathrm{LB}-\mathrm{KH}\left(\mathscr{C}^{\text {parent }}\right)=\mathrm{LB}-\mathrm{KH}\left(\mathscr{C}^{\text {cur }}\right)+1 .
$$

This relation means that $\mathrm{LB}-\mathrm{KH}\left(\mathscr{C}^{\text {cur }}\right)+1$ is a valid lower bound for $\mathscr{C}^{\text {cur }}$ and it always dominates $\mathrm{LB}-\mathrm{N}\left(\mathscr{C}^{\text {cur }}\right)$ from the definition of LB-N. In this case, $\mathrm{LB}-\mathrm{KH}\left(\mathscr{C}^{\text {cur }}\right)+$ 1 can be used as a lower bound instead of LB-N $\left(\mathscr{C}^{\text {cur }}\right)$.

\section{Computational experiments}

In this section we examine the effectiveness of the proposed exact algorithm by computational experiments.

\subsection{Benchmark instances}

We used two sets of benchmark instances in the literature. The first one is the dataset in $[9,3]^{2}$. The instances are characterized by two parameters: the number of stacks $S$ and the number of blocks in every stack $H$. Thus the total number of blocks is $N=S H$, and both $S$ and $H$ range from 3 to 10 . The set contains 40 instances for each combination of $S$ and $H$. The stack height limit $T$ was set to $T:=H+2$. These instances are referred to as CVS instances. The second set derives from [13]. Since the web site of the authors no longer exists, it is mirrored

\footnotetext{
${ }^{2}$ Downloadable from https://www.bwl.uni-hamburg.de/en/iwi/forschung/ projekte/dataprojekte/brp-instances-caserta-etal-2012.zip.
} 
in our web page ${ }^{3}$. The instances in this set are characterized by $S, T$, and $N$. They range from 6 to 10, from 3 to 7 , and from $S(T-1)$ to $S T-1$, respectively. The set contains 100 instances for each combination of $S, T$, and $N$. Among them, 9000 instances with $T \leq 6$ were used because it is too time-consuming to solve all 3500 instances with $T=7$. These instances are referred to as ZQLZ instances.

\subsection{Algorithm specifications}

The proposed exact algorithm was coded in $\mathrm{C}$. The source code is available from our aforementioned web page. The computational experiments were conducted on a desktop computer with an Intel Core i7-6700 3.4GHz CPU and 8GB RAM. The time limit was set to 1800 seconds for each instance.

To describe the exact algorithm, "T", "I", "R", "FB", and "N" are used, which stand for:

T Transitive Relocation Rules A, B, and C (Theorems 1-3) are used,

I Independent Relocation Rule A (Theorem 4) is used,

R Retrieval Rules A and B (Theorems 6 and 7) are used,

FB LB-FB is used as a lower bound,

N LB-N is used as a lower bound.

For example, "FB" is the naive algorithm that employs LB-FB as a lower bound, and "TIR/N" stands for the algorithm that employs LB-N, Transitive Relocation Rules A-C (T), Independent Relocation Rule A (I), and Retrieval Rules A and B (R).

We also improved the exact algorithm in Tanaka and Takii [23] for the restricted BRP with distinct priorities by the dominance rules. In this case, "T" and " $R$ " stand for:

T Transitive Relocation Rule A (Theorem 2) is used.

R Retrieval Rule B (Theorem 7) is used.

\footnotetext{
${ }^{3}$ https://sites.google.com/site/shunjitanaka/brp.
} 


\subsection{Results for CVS instances}

First, the unrestricted BRP is investigated through computational results for the CVS instances. They are summarized in Table 3. In this table, "\%imp" presents the percentages of instances for which the initial lower bound is given not by LB-KH but by LB-KH +1 . For example, LB-FB is equal to $\mathrm{LB}-\mathrm{KH}+1$ for the initial configurations of $0.425 \times 40=17$ instances with $S=6$ and $H=6$, whereas $\mathrm{LB}-\mathrm{N}=\mathrm{LB}-\mathrm{KH}+1$ for all these 40 instances. The difference between LB-FB and LB-N is significant when $S$ is large. LB-FB becomes less efficient as $S$ becomes larger, because the lefthand side of (7) becomes smaller so that (7) is less often satisfied. In contrast, LB-N is not affected by $S$, and it is almost always better than LB-KH when $H \geq 5$. This result verifies the effectiveness of the proposed lower bound LB-N.

In Table 3, the column "opt" denotes the number of instances solved to optimality within the time limit, and "ave" and "max" the average and maximum computation times in seconds, respectively. In addition, the average objective value by the greedy heuristic and the average optimal (or best) value are provided in the columns "HEUR" and "UB", respectively. The results of the exact algorithms in $[17,26]$ are also presented in the table, although only the number of instances solved to optimality is provided in [17]. In [26], several combinations of lower bounds, upper bounds, and branch-and-bound algorithms were tested. For a fair comparison, we picked up the best result for each group of the instances, in terms of the number of instances solved to optimality and the average computation time. Please also note that average computation time in [26] is over instances solved to optimality, in contrast to ours over 40 instances. It is verified from the table that the dominance rules and the new lower bound bring a significant improvement of the exact algorithm. Indeed, the number of instances solved to optimality increases and the computation time is decreased from the naive algorithm FB. The greedy heuristic yields near-optimal solutions for small-sized instances with $H=3$. However, the gap from the optimal value increases as $H$ increases, and it reaches an average of 6.3 relocations for instances with $H=S=6$. It seems difficult to obtain a good solution for such instances by a simple greedy heuristic. With regard to the exact algorithms in the literature, FB already outperforms the $\mathrm{A}^{*}$ algorithm in [17] and yields a similar performance to the branch-and-bound algorithms in [26] (note that a time limit of 24 hours was imposed in [17], in contrast to 1800 seconds in [26] and our case). Since our proposed algorithm TIR/N obviously outperforms FB, we can conclude that TIR/N outperforms the existing algorithms in $[17,26]$.

More detailed results of the proposed exact algorithm are presented in Table 4. 
This table provides the number of instances solved to optimality and the average computation time in seconds with and without the three types of dominance rules, to examine their effects as well as that of the new lower bound. The results in this table indicate that both the dominance rules and the new lower bound yield significant improvements from the naive algorithm FB. It is observed from the results of $\mathrm{T} / \mathrm{N}$ and $\mathrm{I} / \mathrm{N}$ that the independent relocation rule becomes more effective than the transitive relocation rules as the number of stacks increases. This can be explained as follows. The total number of combinations of two relocations is $S^{2}(S-1)^{2}$, and the number of two independent relocations among them is $S(S-$ 1) $(S-2)(S-3)$. The independent relocation rule eliminates half of them at best. On the other hand, the number of transitive relocations is $S^{2}(S-1)$. Even if all of them are eliminated by the transitive relocation rules, the latter are less effective than the former when $S$ is large, because $S(S-1)(S-2)(S-3) / 2>$ $S^{2}(S-1)$. Unlike these two types of rules, the retrieval rules did not greatly affect the efficiency of the algorithm. R/N was even slower than $\mathrm{N}$ for some instances due to computational efforts required for applying the dominance rules. Nevertheless, TIR/N was always a little faster than TI/N. It is because the program codes for checking the dominance rules are mostly composed of common parts. Hence, applying the retrieval rules in addition to the other rules requires only a small computational effort.

Next, the restricted BRP is investigated through computational results for the CVS instances. In Table 5, our previous algorithm in [23] and an improved one TR using the dominance rules are compared. We also present the results of four existing exact approaches: the branch-and-price algorithm [16], the $\mathrm{A}^{*}$ algorithm [17], and the IP approaches [21, 28]. It should be noted that the average computation time in $[21,28]$ is over non-trivial instances solved to optimality: Lower and upper bounds are computed for each instance in pre-processing, and it is considered as a non-trivial instance when the LB/UB gap is not zero. With regard to $[16,17]$, only the number of instances solved to optimality is shown in the table. It is because average computation time in [16] is provided separately for the root node and inner nodes of the search tree, which makes it difficult to evaluate overall computation time. Moreover, computation time is not reported in [17]. Among the four approaches, the IP approach by Galle et al. [28] succeeded in solving more instances to optimality than $[16,17,21]$. Our previous algorithm [23] solved further more instances to optimality in spite of a shorter time limit of 1800 seconds (the time limit in [28] was set to 1 hour). The dominance rules improved it and TR solved nine more instances to optimality. Although the improvement was not so significant as that for the unrestricted BRP, we can see that TR yields the best 
performance among these approaches.

There are several studies on the exact approaches to the restricted BRP whose results are not presented in Table 5. Expósito-Izquierdo et al. [18] corrected a mistake in the ILP formulation in [3]. They also proposed a branch-and-bound algorithm that is considerably faster than their previous $A^{*}$ algorithm [17]. Eskandari and Azari [19] corrected the mistake in the ILP formulation in [3] as well, and introduced some cuts to further improve it. Ku and Arthanari [24] proposed another branch-and-bound algorithm. However, these four approaches were applied to only 5 out of 40 instances in each instance group, so that their results are not included in Table 5. To examine them, computation times by these approaches are summarized in Tables 6 and 7. We also present, in the same tables, computation times by our previous algorithm [23], TR in this study, and the IP approach [28]. Among the approaches in [18, 19, 24, 28], the branch-and-bound algorithm [18] seems to be the fastest for most of the instances in Table 6, although computation times by the IP approach [28] are available only for non-trivial instances (not marked with an asterisk). For the instances in Table 7, only results by the two IP approaches [19, 28] are available, and we can see that [28] is faster than [19]. On the other hand, our previous algorithm and TR took less than 0.01 seconds for every instance in these tables, thus outperforming the other approaches in terms of computation time.

\subsection{Results for ZQLZ instances}

In Table 8, results for the ZQLZ instances are summarized. The number of instances is shown in the column " $n$ ". We can again verify that the dominance rules and the new lower bound considerably improve the efficiency of the exact algorithms. Unlike the CVS instances, the ZQLZ instances seem to be easier to solve as restricted BRPs than as unrestricted BRPs, regardless of $S$. The reason for it is not clear, but the two sets are different in the fill rate: $2 S$ slots are not filled with blocks in the CVS instances $(T=H+2)$, whereas at most $S$ slots are not filled in the ZQLZ instances. This difference could have affected the efficiency of the exact algorithms.

Zhu et al. [13] proposed IDA* algorithms for the restricted and unrestricted BRPs. With regard to the restricted BRP, our previous algorithm [23] outperforms their algorithm, as already reported in [23]. However, the comparison is not easy on the unrestricted BRP only from limited information in [13]. Nevertheless, we try to draw as fair a comparison as possible. According to the detailed results obtained from the authors' web page before it was closed, their algorithm IDA*U solved 5307 instances out of 9000 to optimality, when the maximum number 
of nodes generated in the search tree was limited to $2^{30}$. They also proposed a better algorithm named IDA*-UM that employs a memory dominance technique to eliminate the same configurations in the search tree. Unfortunately, they imposed a very short time limit of 1 second on it in order to use it not as an exact algorithm but as a metaheuristic. According to the results presented in [13], IDA*-UM solved 4868 instances to optimality within this time limit. On the other hand, TIR/N solved to optimality 8135 instances when the maximum number of generated nodes was limited to $2^{30}$, and 5472 instances within 0.01 seconds. Although the Pentium4 3.0GHz CPU in [13] is considerably slower than ours, we can conclude from these facts that our proposed algorithm outperforms IDA*-U and IDA*-UM in [13].

\section{Conclusion}

In this paper we proposed an exact algorithm for the unrestricted BRP with distinct priorities. For this purpose, we derived three types of dominance properties to eliminate unnecessary nodes in the search tree. We also improved the lower bound by Forster and Bortfeldt [12]. These enabled us to solve a larger number of benchmark instances to optimality than the existing exact algorithms in the literature. Some of the dominance properties are applicable also to the restricted BRP, and they contributed to improving our previous exact algorithm in [23] for this class of problem. Now, we can efficiently solve to optimality the unrestricted and restricted BRPs with distinct priorities. However, to the best of the authors' knowledge, no good exact algorithm exists for the unrestricted BRP with duplicate priorities. To handle duplicate priorities, the proposed dominance properties should be modified. It might be possible to derive specific properties to this class

of problem. The proposed lower bound should also be extended. These topics are left for future research.

\section{Acknowledgements}

This work was supported by JSPS KAKENHI Grant Number JP15K01187.

\section{References}

[1] Lehnfeld J, Knust S. Loading, unloading and premarshalling of stacks in storage areas: Survey and classification. Eur J Oper Res 2014;239:297-312. 
[2] Blasum U, Bussieck MR, Hochstättler W, Moll C, Scheel H-H, Winter T. Scheduling trams in the morning. Math Methods Oper Res 1999;49:137-148.

[3] Caserta M, Schwarze S, Voß S. A mathematical formulation and complexity considerations for the blocks relocation problem. Eur J Oper Res 2012;219:96-104.

[4] König FG, Lübbecke M, Möhring R, Schäfer G, Spenke I. Solutions to real-world instances of PSPACE-complete stacking. Lect Notes Comput Sci 2007;4698:729-740.

[5] Kim KH, Hong G-P. A heuristic rule for relocating blocks. Comput Oper Res 2006;33:940-954.

[6] Wan Y-w, Liu J, Tsai P-C. The assignment of storage locations to containers for a container stack. Nav Res Logist 2009;56:699-713.

[7] Caserta M, Schwarze S, Voß S. A new binary description of the blocks relocation problem and benefits in a look ahead heuristic. Lect Notes Comput Sci 2009;5482:37-48.

[8] Caserta M, Voß S. Corridor selection and fine tuning for the corridor method. Lect Notes Comput Sci 2009;5851:163-175.

[9] Caserta M, Voß S, Sniedovich M. Applying the corridor method to a blocks relocation problem. OR Spectr 2011;33:915-929.

[10] Lee Y, Lee Y-J. A heuristic for retrieving containers from a yard. Comput Oper Res 2010;37:1139-1147.

[11] Ünlüyurt T, Aydın C. Improved rehandling strategies for the container retrieval process. J Adv Transp 2012;46:378-393.

[12] Forster F, Bortfeldt A. A tree search procedure for the container relocation problem. Comput Oper Res 2012;39:299-309.

[13] Zhu W, Qin H, Lim A, Zhang H. Iterative deepening $A^{*}$ algorithms for the container relocation problem. IEEE Trans Autom Sci Eng 2012;9:710-722.

[14] Petering MEH, Hussein MI. A new mixed integer program and extended look-ahead heuristic algorithm for the block relocation problem. Eur J Oper Res 2013;231:120-130. 
[15] Jovanovic R, Voß S. A chain heuristic for the Blocks Relocation Problem. Comput Ind Eng 2014;75:79-86.

[16] Zehendner E, Feillet D. A branch and price approach for the container relocation problem. Int J Prod Res 2014;52:7159-7176.

[17] Expósito-Izquierdo C, Melián-Batista B, Moreno-Vega JM. A domainspecific knowledge-based heuristic for the Blocks Relocation Problem. Adv Eng Infor 2014;28:327-343.

[18] Expósito-Izquierdo C, Melián-Batista B, Moreno-Vega JM. An exact approach for the Blocks Relocation Problem. Expert Syst Appl 2015;42:64086422.

[19] Eskandari H, Azari E. Notes on mathematical formulation and complexity considerations for blocks relocation problem. Scientia Iranica. Transaction E: Industrial Engineering 2015;22(6):2722-2728.

[20] Jin B, Zhu W, Lim A. Solving the container relocation problem by an improved greedy look-ahead heuristic. Eur J Oper Res 2015;240:837-847.

[21] Zehendner E, Caserta M, Feillet D, Schwarze S, Voß S. An improved mathematical formulation for the blocks relocation problem. Eur J Oper Res 2015;245:415-422.

[22] Lin D-Y, Lee Y-J, Lee Y. The container retrieval problem with respect to relocation. Transp Res Part C 2015;52:132-143.

[23] Tanaka S, Takii K. A faster branch-and-bound algorithm for the block relocation problem. IEEE Trans Autom Sci Eng 2016;13:181-190.

[24] Ku D, Arthanari TS. On the abstraction method for the container relocation problem. Comput Oper Res 2016;68:110-122.

[25] Zhang R, Liu S, Kopfer H. Tree search procedures for the blocks relocation problem with batch moves. Flex Serv Manuf J 2016;28:397-424.

[26] Tricoire F, Scagnetti J, Beham A. New insights on the block relocation problem. Comput Oper Res 2018;89:127-139. 
[27] de Melo da Silva M, Erdoğan G, Battarra M, Strusevich V. The Block Retrieval Problem. Eur J Oper Res 2017. available online. DOI: 10.1016/j.ejor.2017.08.048

[28] Galle V, Barnhart C, Jaillet P. A new binary formulation of the restricted container relocation problem based on a binary encoding of configurations, Eur J Oper Res 2017. available online. DOI: 10.1016/j.ejor.2017.11.053

[29] Akyüz MH, Lee C-Y. A mathematical formulation and efficient heuristics for the dynamic container relocation problem. Nav Res Logist 2014;61:101-118.

[30] Zehendner E, Feillet D, Jaillet P. An algorithm with performance guarantee for the Online Container Relocation Problem. Eur J Oper Res 2017;259:4862 .

[31] Ku D, Arthanari TS. Container relocation problem with time windows for container departure. Eur J Oper Res 2016;252:1031-1039.

[32] Avriel M, Penn M. Exact and approximate solutions of the container ship stowage problem. Comput Ind Eng 1993;25:271-274.

[33] Avriel M, Penn M, Shpirer N, Witteboon S. Stowage planning for container ships to reduce the number of shifts. Ann Oper Res 1998;76:55-71.

[34] Avriel M, Penn M, Shpirer N. Container ship stowage problem: complexity and connection to the coloring of circle graphs. Discrete Appl Math 2000;103:271-279.

[35] Dubrovsky O, Levitin G, Penn M. A genetic algorithm with a compact solution encoding for the container ship stowage problem. J Heuristics 2002;8:585-599.

[36] Tierney K, Pacino D, Jensen RM. On the complexity of container stowage planning problems. Discrete Appl Math 2014;169:225-230.

[37] Wang N, Zhang Z, Lim A. The stowage stack minimization problem with zero rehandle constraint. Lect Notes Comput Sci 2014;8482:456-465.

[38] Tang L, Liu J, Rong A, Yang Z. An effective heuristic algorithm to minimise stack shuffles in selecting steel slabs from the slab yard for heating and rolling. J Oper Res Soc 2001;52:1091-1097. 
[39] Tang L, Liu J, Rong A, Yang Z. Modelling and a genetic algorithm solution for the slab stack shuffling problem when implementing steel rolling schedules. Int J Prod Res 2002;40:1583-1595.

[40] Singh KA, Srinivas, Tiwari SMK. Modelling the slab stack shuffling problem in developing steel rolling schedules and its solution using improved Parallel Genetic Algorithms. Int J Prod Econ 2004;91:135-147.

[41] Tang L, Ren H. Modelling and a segmented dynamic programming-based heuristic approach for the slab stack shuffling problem. Comput Oper Res 2010;37:368-375.

[42] Cheng X, Tang L. A scatter search algorithm for the slab stack shuffling problem. Lect Notes Comput Sci 2010;6145:382-389.

[43] Kim B-I, Koo J, Sambhajirao HP. A simplified steel plate stacking problem. Int J Prod Res 2011;49:5133-5151.

[44] Tang L, Zhao R, Liu J. Models and algorithms for shuffling problems in steel plants. Nav Res Logist 2012;59:502-524.

[45] Lee Y, Hsu N-Y. An optimization model for the container pre-marshalling problem. Comput Oper Res 2007;34:3295-3313.

[46] Lee Y, Chao S-L. A neighborhood search heuristic for pre-marshalling export containers. Eur J Oper Res 2009;196:468-475.

[47] Caserta M, Voß S. A corridor method-based algorithm for the premarshalling problem. Lect Notes Comput Sci 2009;5484:788-797.

[48] Choe R, Park T, Oh M-S, Kang J, Ryu KR. Generating a rehandling-free intra-block remarshaling plan for an automated container yard. J Intell Manuf 2011;22:201-217.

[49] Huang S-H, Lin T-H. Heuristic algorithms for container pre-marshalling problems. Comput Ind Eng 2012;62:13-20.

[50] Bortfeldt A, Forster F. A tree search procedure for the container premarshalling problem. Eur J Oper Res 2012;217:531-540. 
[51] Expósito-Izquierdo C, Melián-Batista B, Moreno-Vega M. Pre-Marshalling Problem: Heuristic solution method and instances generator. Expert Syst Appl 2012;39:8337-8349.

[52] Voß S. Extended mis-overlay calculation for pre-marshalling containers. Lect Notes Comput Sci 2012;7555:86-91.

[53] van Brink $M$ van der Zwaan R. A branch and price procedure for the container premarshalling problem. Lect Notes Comput Sci 2014;8737:798-809.

[54] Wang N, Jin B, Lim A. Target-guided algorithms for the container premarshalling problem. Omega 2015;53:67-77.

[55] Zhang R, Jiang Z-Z, Yun WY. Stack pre-marshalling problem: A heuristicguided branch-and-bound algorithm. Int J Ind Eng 2015;22:509-523.

[56] Hottung A, Tierney K. A biased random-key genetic algorithm for the container pre-marshalling problem. Comput Oper Res 2016;75:83-102.

[57] Wang N, Jin B, Zhang Z, Lim A. A feasibility-based heuristic for the container pre-marshalling problem. Eur J Oper Res 2017;256:90-101.

[58] Jovanovic R, Tuba M, Voß S. A multi-heuristic approach for solving the pre-marshalling problem. Cent Europ J Oper Re 2017;25:1-28.

[59] Tierney K, Pacino D, Voß S. Solving the pre-marshalling problem to optimality with A* and IDA*. Flex Serv Manuf J 2017;29:223-259.

[60] Gupta N, Nau DS. On the complexity of blocks-world planning. Artif Intell 1992;56:223-254.

[61] Slaney J, Thiébaux S. Blocks World revisited. Artif Intell 2001;125:119_ 153. 


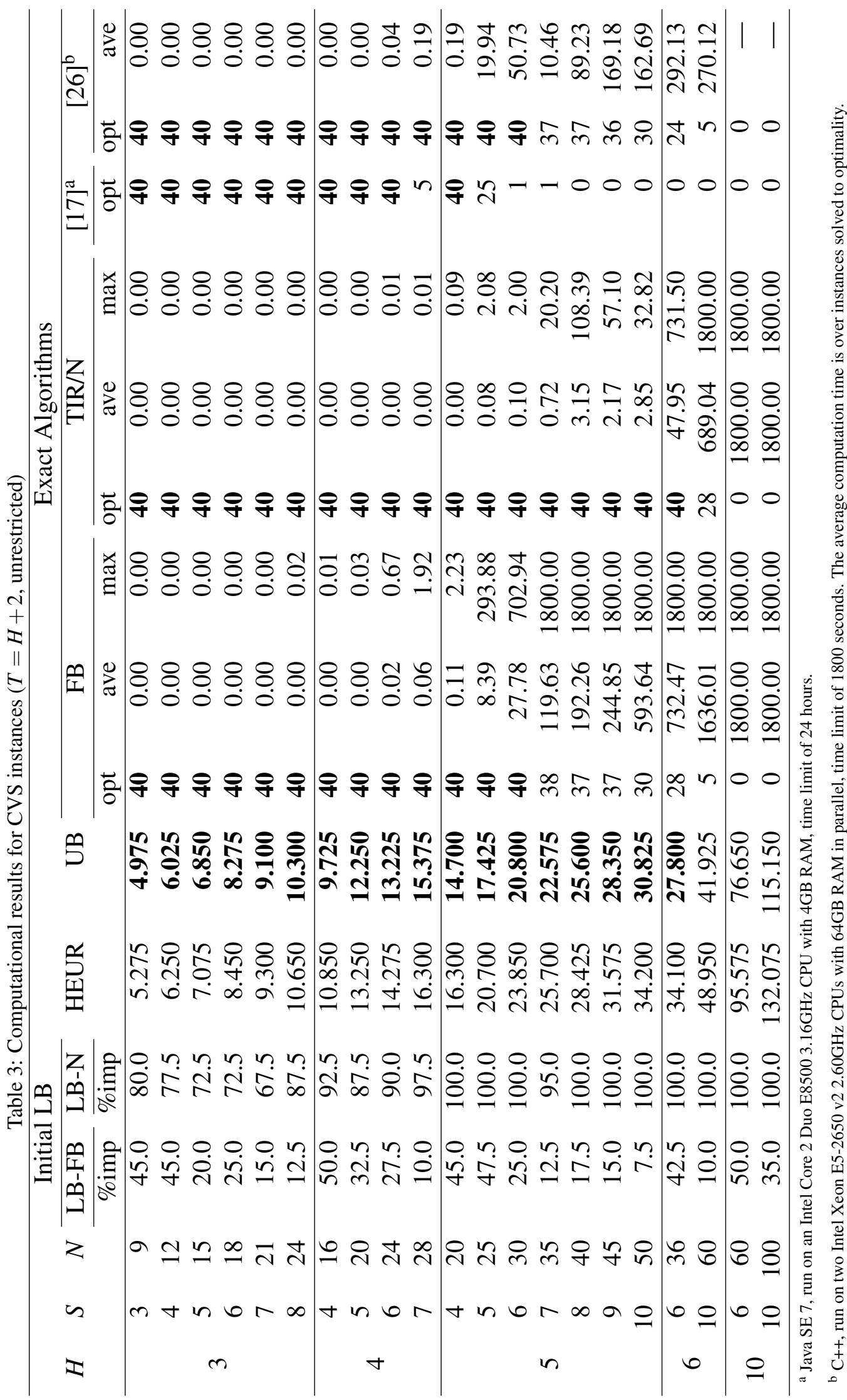




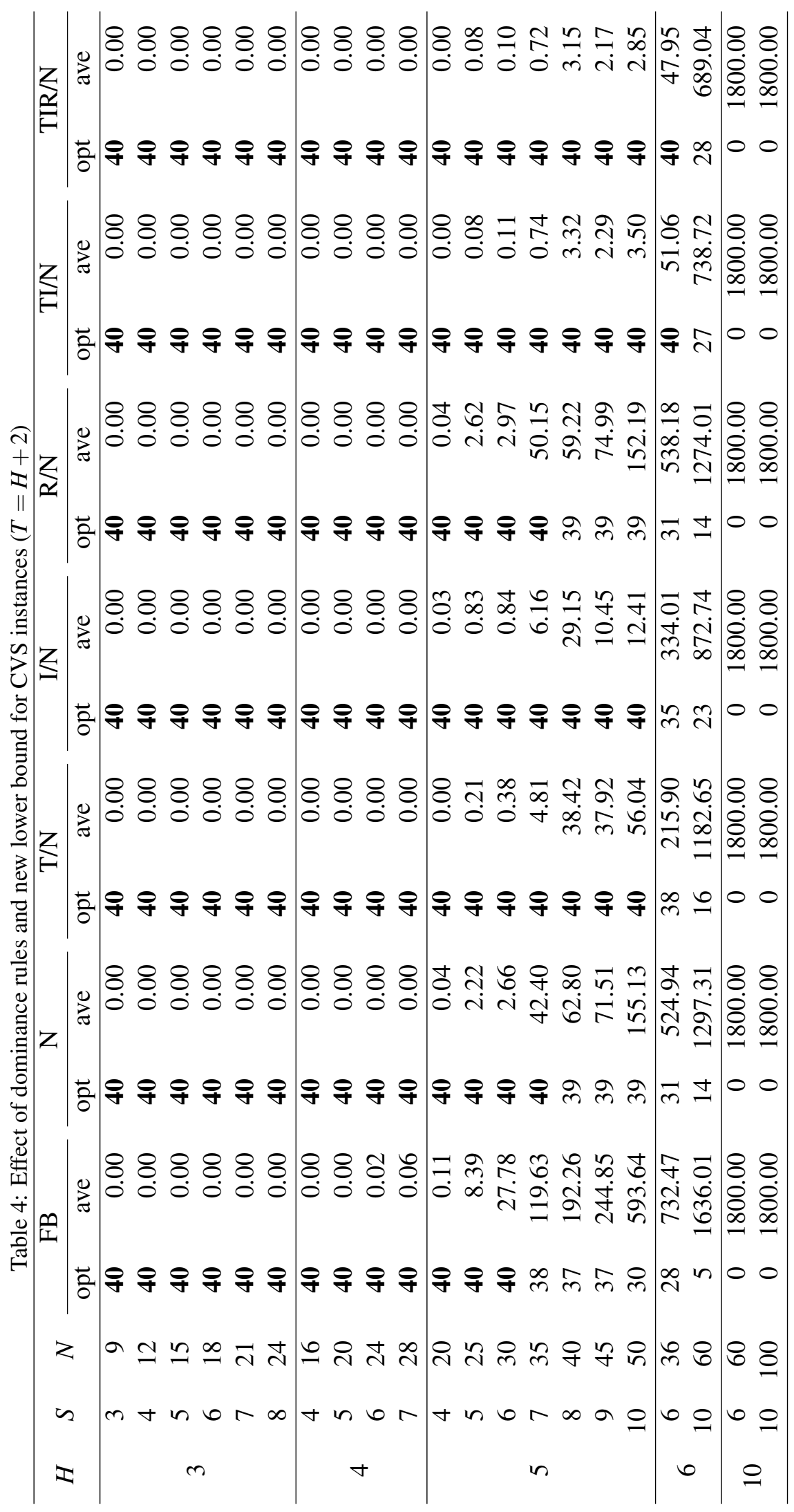




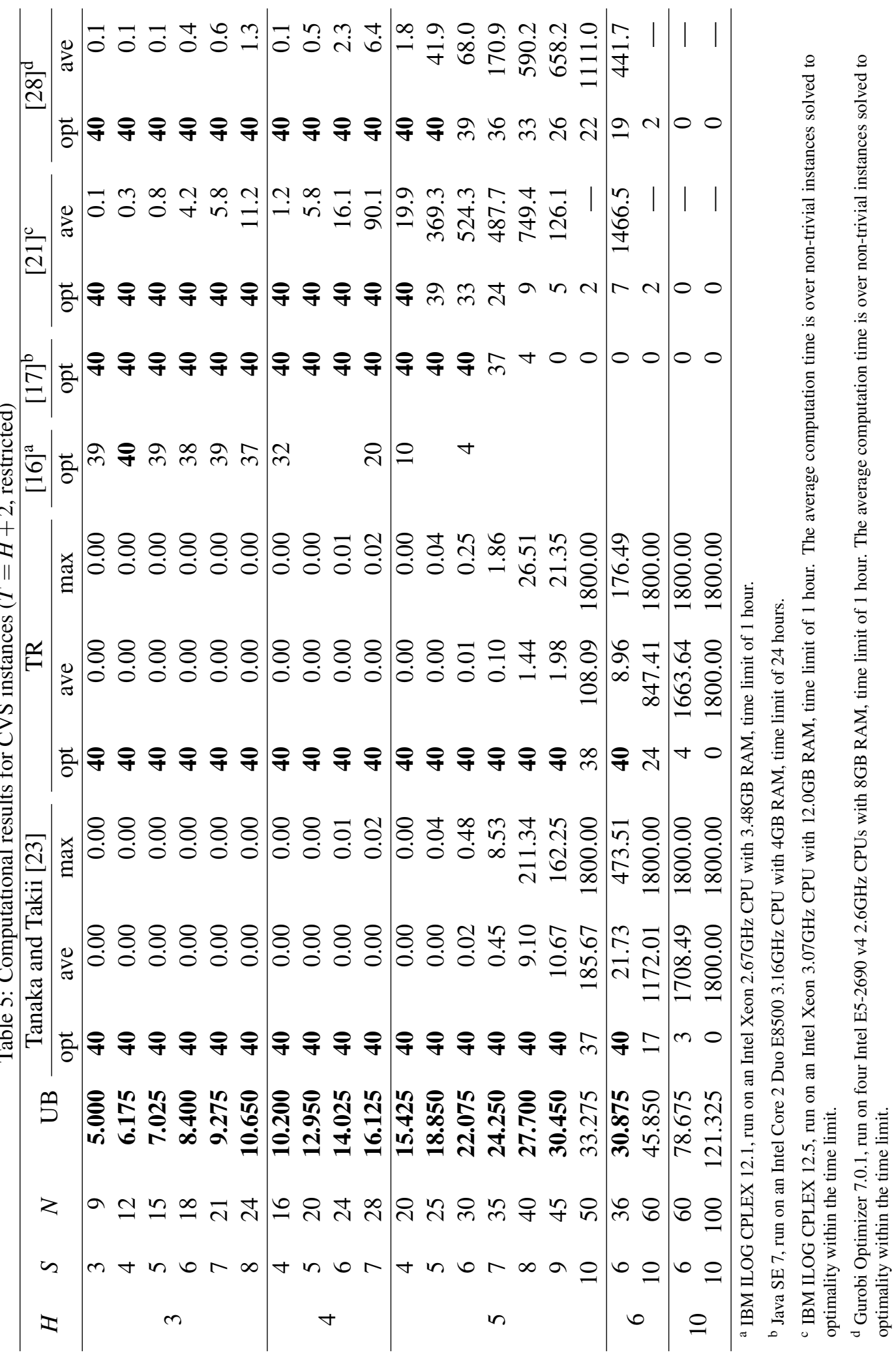


Table 6: Comparison of computation time (in seconds) for a subset of CVS instances: Our previous branch-and-bound algorithm in [23], TR in this study, IP approach and b\&b algorithm in [18], IP approach in [19], b\&b algorithm in [24], and IP approach in [28] ( $T=H+2$, restricted)

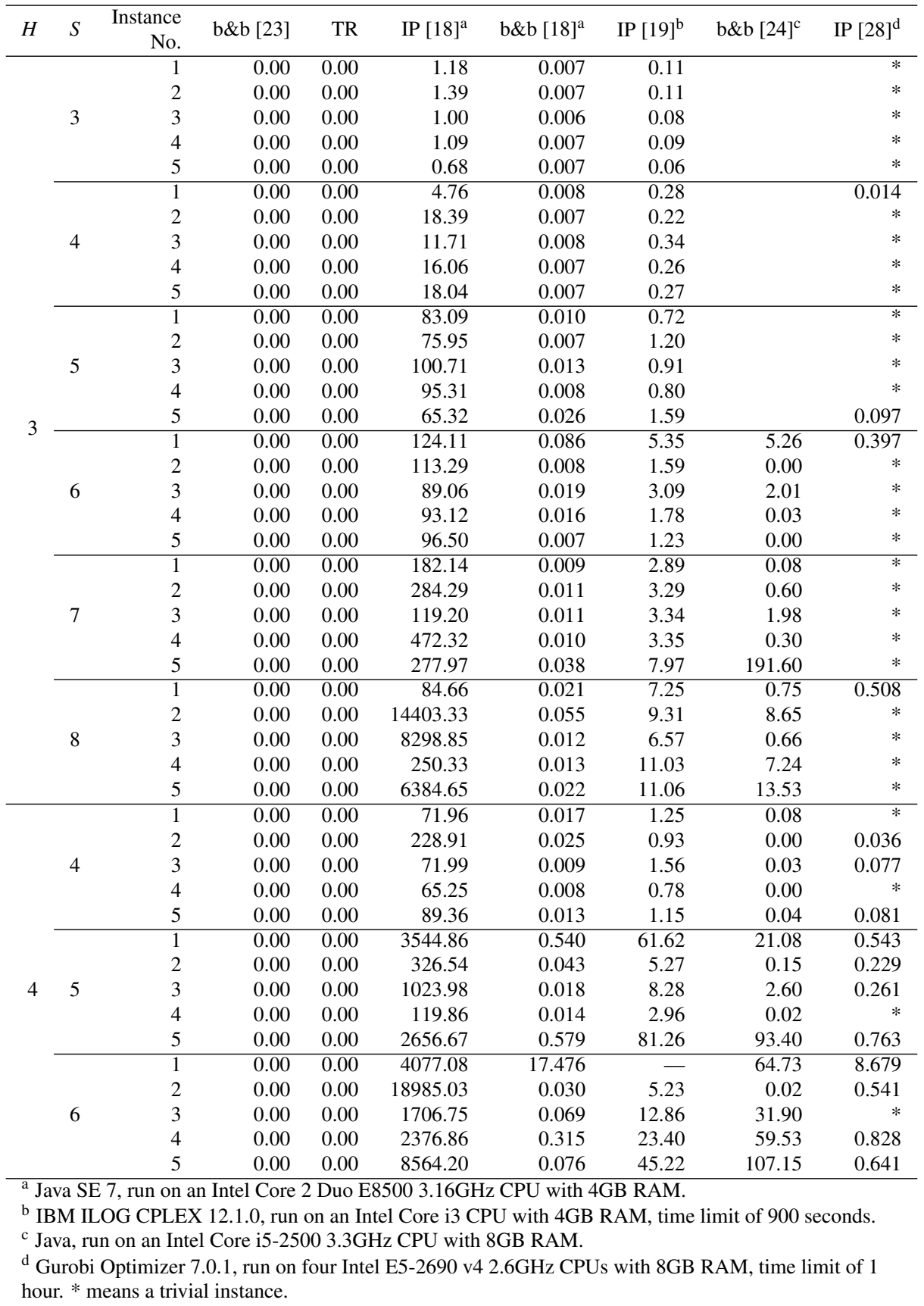


Table 7: Comparison of computation time (in seconds) for a subset of CVS instances: Our previous branch-and-bound algorithm in [23], TR in this study, and IP approaches in $[19,28](T=H+2$, restricted)

\begin{tabular}{|c|c|c|c|c|c|c|}
\hline$H$ & $S$ & $\begin{array}{r}\text { Instance } \\
\text { No. }\end{array}$ & b\&b [23] & $\mathrm{TR}$ & IP [19] & $\operatorname{IP}[28]^{\mathrm{d}}$ \\
\hline \multirow{5}{*}{4} & \multirow{5}{*}{7} & 1 & 0.00 & 0.00 & 44.10 & 2.995 \\
\hline & & 2 & 0.00 & 0.00 & 20.47 & 1.245 \\
\hline & & 3 & 0.00 & 0.00 & 18.95 & * \\
\hline & & 4 & 0.00 & 0.00 & 49.78 & 2.310 \\
\hline & & 5 & 0.00 & 0.00 & 85.75 & 2.160 \\
\hline \multirow{10}{*}{5} & \multirow{5}{*}{4} & 1 & 0.00 & 0.00 & 118.20 & 0.295 \\
\hline & & 2 & 0.00 & 0.00 & 185.36 & 1.060 \\
\hline & & 3 & 0.00 & 0.00 & 5.90 & 0.320 \\
\hline & & 4 & 0.00 & 0.00 & 5.01 & 0.170 \\
\hline & & 5 & 0.00 & 0.00 & 118.16 & 0.652 \\
\hline & \multirow{5}{*}{5} & 1 & 0.00 & 0.00 & - & 6.079 \\
\hline & & 2 & 0.00 & 0.00 & 22.06 & 0.422 \\
\hline & & 3 & 0.00 & 0.00 & - & 25.542 \\
\hline & & 4 & 0.00 & 0.00 & - & 5.538 \\
\hline & & 5 & 0.00 & 0.00 & 46.30 & 0.837 \\
\hline
\end{tabular}

${ }^{\mathrm{b}}$ IBM ILOG CPLEX 12.1.0, run on an Intel Core i3 CPU with 4GB RAM, time limit of 900 seconds.

${ }^{\mathrm{d}}$ Gurobi Optimizer 7.0.1, run on four Intel E5-2690 v4 2.6GHz CPUs with 8GB RAM, time limit of 1 hour. * means a trivial instance. 


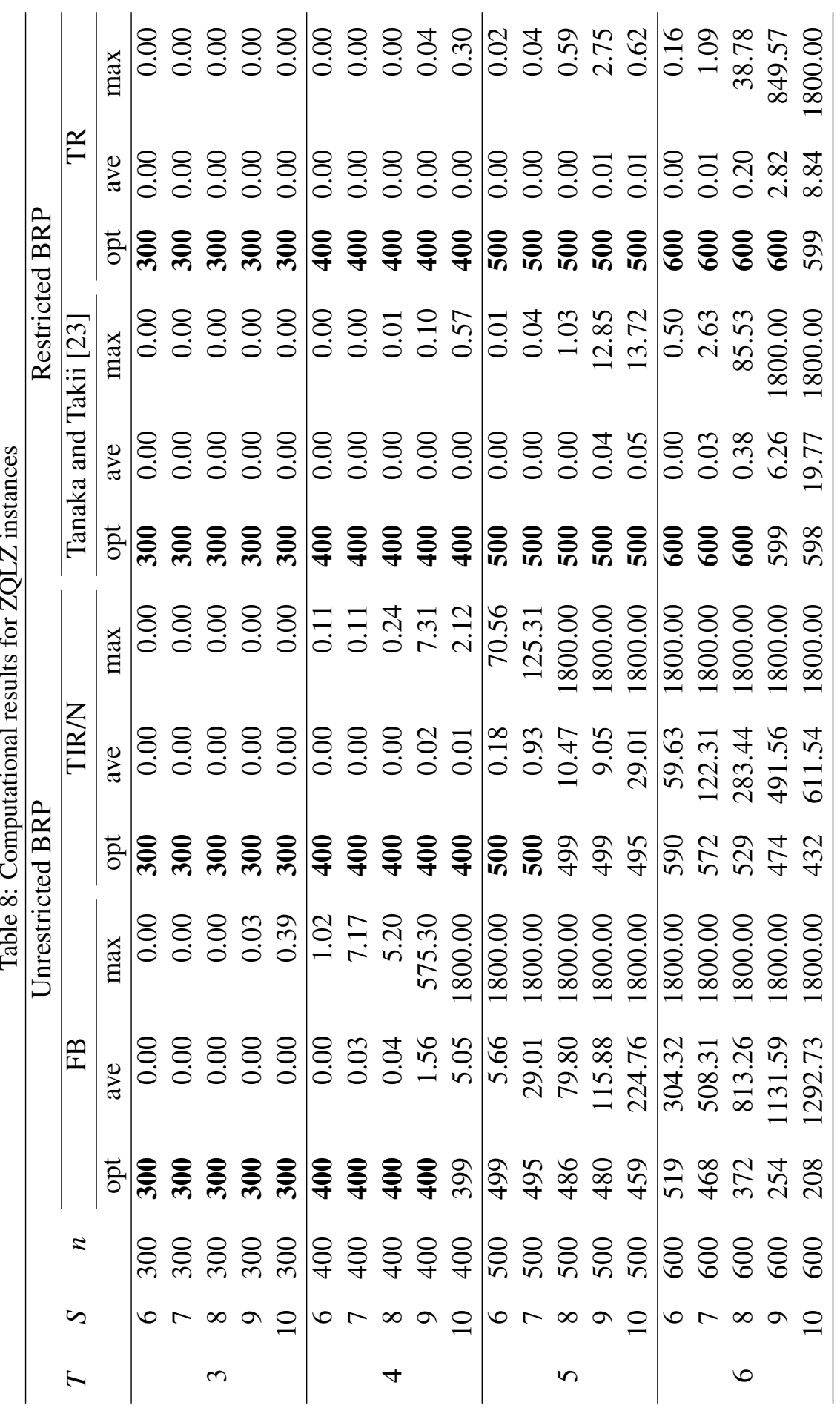

